\title{
Dirlis Messner
}

\section{Fallstricke nud Grenzen der Natzwerkstenerung}

Seit Anfang der 80er Jahre wird die Beschäftigung mit Politiknetzwerken zu einer Metapher für die Auseinandersetzung mit gesellschaftlichen Organisationsmustern und politischen Steuerungsmechanismen, die mit den klassischen staatstheoretischen Ansätzen, basierend auf den Prämissen der klaren Trennung von Staat und Gesellschaft und der inneren und äußeren Souveränität des Staates, nicht thematisiert werden können. Netzwerke erscheinen zuweilen als Organisationsstrukturen, die den zunehmenden Verlust nationalstaatlicher Steuerungsfähigkeit zu kompensieren vermögen. Im Zentrum dieser steuerungstheoretischen Konzepte steht die Beobachtung, daß moderne Gesellschaften in vielen Bereichen durch netzwerkartige Organisationsformen gekennzeichnet sind und Problemlösungen zunehmend auf »horizontal policy coordination《 in »joint decision systems« oder »pluralistischen policy Netzwerken« basieren (Powell 1990; Marin/Mayntz 1991; Héritier 1993). Staatliche Institutionen verfügen in einer Vielzahl von Politikfeldern, in der Bildungs- und Forschungspolitik, der Industrie- und Technologiepolitik, der Regional- oder auch der Umweltpolitik, längst nicht mehr über die notwendigen Steuerungsressourcen (z. B. Wissen über komplexe Wirkungszusammenhänge in den jeweiligen Bereichen, Implementationsfähigkeit, Kontrollkapazität), um unabhängig von den gesellschaftlichen Akteuren Politikgestaltung zu betreiben. Andersherum sind auch die gesellschaftlichen Akteure auf die Kooperation mit öffentlichen Institutionen angewiesen. Am Beispiel von Technologieund Industriepolitiken heißt dies: Einerseits verliert der Staat angesichts der Globalisierung der Ökonomie und der Komplexität industrieller Produktion an Steuerungsfähigkeit in der Wirtschaftspolitik. Andererseits bleiben die Unternehmen auf leistungsfähige Wirtschaftsstandorte, Wissenschaftssysteme, Institutionengefüge und langfristig stabile Erwartungshorizonte angewiesen (Eßer et al. 1994). Angesichts dieser wechselseitigen Abhängigkeit zwischen Unternehmen, öffentlichen Institutionen und gesellschaftlichen Akteuren entsteht ein wirtschaftspolitisches Aktionsfeld, in dem weder der Markt (also die dezentral agierenden Unternehmen), noch der hierarchisch steuernde Staat erfolgreich sein können. 
Insgesamt zeichnet sich in vielen gesellschaftlichen Bereichen ab, daß der Autonomieverlust des Staates sich nicht automatisch in einem Autonomiegewinn für gesellschaftliche Akteursgruppen niederschlägt. Es entsteht eine wechselseitige Abhängigkeit, die dazu führt, daß Politikgestaltung zunehmend in formellen oder »informel political infrastructures outside conventional channels such as legislative, executive and administrative organizations « (Kenis/Schneider 1991, 27) vorbereitet wird - Politiknetzwerke gewinnen an Bedeutung. Je nach Sichtweise kommt es angesichts dieser interdependenten Strukturen zwischen Staat und gesellschaftlichen Akteuren zu einer »Vergesellschaftung der Politik oder auch einer »Politisierung oder Verstaatlichung der Gesellschaft $\ll$.

Die netzwerkartigen Organisationsformen und eine darauf basierende politische Steuerung können demnach als Reaktionen auf soziale, politische und ökonomische Ausdifferenzierungen, Spezialisierungen und Interdependenzen interpretiert werden. Netzwerkkonzepte konvergieren in einem wichtigen Punkt: Die Koordination zwischen politischen, sozialen und ökonomischen Akteuren und darauf basierende Problemlösungsformen in »network-like forms of governance « unterscheiden sich von den drei Standardformen gesellschaftlicher Koordination in modernen Gesellschaften, als da wären

- der Preismechanismus, der das Verhalten von Unternehmen und Konsumenten in anonymen Märkten koordiniert;

- der hierarchisch-majoritäre Politikmodus, der vorsieht, daß die durch demokratische Wahlen legitimierte Parlamentsmehrheit oder die durch diese gestützte Exekutive auf der Grundlage von Rechtsetzungs- und Steuerungskompetenz für das Gemeinwesen verbindliche Entscheidungen herbeiführt und umsetzt;

- die hierarchisch übergeordnete Instanz, die (innerhalb staatlicher Institutionen, nicht-staatlicher Organisationen sowie von Unternehmen) für die untergeordneten Einheiten verbindliche Entscheidungen trifft (hierarchische Entscheidungs- und Problemlösungsmechanismen).

Scharpf (1992b, 51) umschreibt diesen Sachverhalt wie folgt:

»Markt und Hierarchie gelten gemeinhin als die wichtigsten Koordinationsmechanismen in modernen Gesellschaften. Aber selbst in der privaten Wirtschaft kommen viele wichtige Austausch- und Kooperationsbeziehungen durch Verhandlungen zustande, deren Ergebnis nicht durch Marktpreise bestimmt wird; umgekehrt stellen sich viele Interaktionen in formal hierarchischen Organisationen faktisch als (möglicherweise asymmetrische) Verhandlungen zwischen Partnern mit eigenen Interessen und Handlungsspielräumen dar; und ebenso oft erweist sich die Formulierung und Implementation staaticher Politik eher als Ergebnis multilateraler Verhandlungen zwischen einer Vielzahl staatlicher und außerstaatlicher Akteure denn als einseitig-hierarchische Steuerungsintervention eines monolithischen Staates.« 
Der Begriff »Netzwerk« betont die Selbstorganisation bzw. -Koordination zwischen de facto autonomen Akteuren zur Erreichung eines gemeinsamen Resultates. Kenis/Schneider sehen in den Netzwerkansätzen gar ein neues Paradigma zum Verständnis der »architecture of complexity « (Kenis/ Schneider 1991, 25), das von seiner Bedeutung her vergleichbar sein könnte mit dem zuvor dominanten Konzept der Hierarchie als Instrument der Komplexitätsreduktion in sich stets weiter ausdifferenzierenden Gesellschaften (Simon 1973):

»Policy networks are mechanisms of political ressource mobilization in situations where the capacity for decision making, program formulation and implementation is widely distributed or dispursed among private and public actors ... In situations where policy resources are dispersed and context (or actor) dependent, a network is the only mechanism to mobilize and pool ressources.« (Kenis/Schneider 1991, 41f)

Die Stärken von Netzwerken werden in einer Vielzahl von insbesondere empiririschen Arbeiten breit diskutiert. Politiknetzwerke können zu einer Entlastung des Staates führen, eine höhere Informiertheit aller Netzwerkakteure und somit ein höheres $\mathrm{Ma} 3$ an »Steuerungsintelligenz « fördern, die Legitimität staatlicher Entscheidungen stärken und zur Bündelung gesellschaftlicher Kreativitäts- und Problemlösungspotentiale beitragen.

In dem vorliegenden Beitrag werden, ohne die Steuerungspotentiale von Netzwerkstrukturen verkennen zu wollen, die Fallstricke und Grenzen der Netzwerksteuerung thematisiert. Diese finden in der breiten Netzwerkliteratur bisher nur wenig Berïcksichtigung, so daß sich die folgende Auseinandersetzung mit den Problemen der Netzwerksteuerung auch als Kritik an affirmativen Netzwerkansätzen lesen läßt. Daß ohne den Steuerungsmechanismus »Markt « komplexe Gesellschaften und Ökonomien, zumal im Weltmaßstab, nicht überlebensfähig sind (Altvater 1993, 11; Altvater 1991), Marktallokation dennoch kein Allheilmittel darstellt, ist in der Literatur zum »Marktversagen« breit dokumentiert. ${ }^{1}$ Ähnliches gilt für den

In der umfangreichen Literatur zurn Marktversagen (vgl. zum Überblick v. Hayek (1952), de Vroey (1991), Kirsch et al. (1982)) können fünf Kernprobleme ausgemacht werden. Erstens kann der Markt dann zu einer unvollkommenen Allokation der Ressourcen führen, wenn es einzelnen Marktteilnehmern möglich ist, aufgrund ihrer Machtposition anderen Kompensationen für negative externe Effekte nicht zu zahlen oder sich für nicht oder nur unzureichende Leistungen entlohnen zu lassen (unvollkommener Wettbewerb). Zweitens kann die Allokation über den Markt prinzipiell nicht exfolgen, wenn private Eigentumsrechte an den als Folge einer Entscheidung enstandenen Zielverwirklichungen nicht geltend gemacht werden können (Nichtgeltung des Ausschlußprinzips). An die Stelle des nichtexistierendeen Marktes muß dann die Allgemeinheit treten. Marktversagen entsteht drittens, wenn die Nutzung eines Gutes durch einen Konsumenten nicht zur Reduzierung des Nutzens führt, den ein anderer Konsument aus dem Gut zu ziehen vermag (nichtrivalisierender Konsum). Viertens ist selbst für den Fall, daß die ersten drei Problemfelder ausgeschlossen werden können, der Markt als Allokationsmechanismus nur auf solche Güter anwendbar, deren Werte in Preisen ausgedrückt werden können, was z. $B$. bei der Beurteilung von Umweltgitern 
Staat, der zweifellos wichtige Steuerungsaufgaben zu erfüllen hat; dennoch gibt es Fälle von »Staatsversagen ${ }^{2}{ }^{2}$ Netzwerkförmige Organisationsmuster und darauf basierende Steuerungsleistungen sind in diesem Sinne keine Ausnahme. Die in der Struktur und Funktionslogik von Netzwerken angelegten Formen von Netzwerkversagen sollen im folgenden herausgearbeitet werden.

Um Mißverständnissen vorzubeugen, sei darauf hingewiesen, daß in einigen der folgenden sieben Problemdimensionen ähnlich gelagerte Kernprobleme der Netzwerkorganisation auftauchen. Die Mehrfachnennung eines Problemes darf nicht mit vermeidbarer Redundanz verwechselt werden, da einige Kernprobleme durch je unterschiedliche Funktionslogiken in jeweiligen Problemdimensionen ausgelöst werden. Erst das genaue Verständnis der Ursachen bestimmter Problemkonstellationen der Netzwerksteuerung erlaubt es, uber Strategien zu deren Vermeidung oder Reduzierung systematisch nachzudenken.

\section{Pas Problem der großen Zahl}

Das erste Problemfeld ist trivial und wird dennoch oft vernachlässigt. Die Schwierigkeiten ausgehandelter Koordination in Netzwerken nehmen mit der Zahl der beteiligten Akteure und ihren interdependeten Handlungsoptionen zu. Scharpf (1993c) verweist darauf, daß in der Theorie kooperativer

außerordentlich schwierig, oft unmöglich ist. Diese vier Kernprobleme werden in aller Regel auch von traditionellen, (neo)-liberalen Ökonomen geteilt. Zudem sorgt der Markt fünftens zwar - mit den skizzierten Einschränkungen - für ein Angebot an Gütern, nicht jedoch für Verteilungsgerechtigkeit. Hier müssen, wie auch der liberale Ralf Dahrendorf (1992. 35) einräumt, "Anrechte« geschaffer werden: "Wenn nicht traditionelle Anrechtsstrukturen gebrochen und Elemente einer Bürgergesellschaft geschaffen werden, bedeutct makroökonomisches Wachstum wenig für die vielen, so sehr der Internationale Währungsfond sich über die Globalstatistik freuen mag.« Der Markt versagt also, wenn es um die Schaffung von Anrechtsveränderungen (Zugang zu Bildungsinstitutionen, Verteilungspolitik usw.) und somit um die gerechte Zuteilung von Lebenschancen geht. Sechstens ist der Markt nicht nur ein Allokationsmechanismus, sondern, wie bereits Marx (1981) und später insbesondere auch Polanyi (1978) gezeigt haben, ein »soziales Verhältnis«. Der Markt ist ohne die Einbettung in spezifische institutionelle Landschaften und deren Verankerung in Wertemusterm der gesellschaftlichen Akteure nicht leistungsfähig. Der Markt ist daher auch nicht, wie z. B. nach 15 Jahren neoliberaler Strukturanpassung mehr als deutlich wird, in Entwicklungsländern oder auch in osteuropäischen Transformationsländern »von oben« oder im Handstreich zu "installieren. Vgl, zur Fragestellung der »nicht-ökonomischen« Voraussetzungen für die Funktionsfähigkeit von Märkten Altvater (1991), Semmler (1991), Eßer et al. (1993 und 1994).

2 Vgl. zu dem Problemfeld Staats- bzw. Bürokratieversagen Altvater (1992), aus institutionenökonomischer Sicht Brücker (1993, 32 ff). Dahrendorf $(1992,33)$ entwickelt die im Prinzip richtige Sichtweise auf das Problem von Staats- und Marktversagen: »Märkte versagen, wenn es um Anrechtsveränderungen geht, und Regierungen versagen (als »Unternehmer«, D.M.) bei der Steigerung des Angebotes, aber es wäre falsch, den Markt oder den Staat für das verantwortlich zu machen, was sie ihrer Natur nach nicht leisten können.« Gleiches gilt natürlich für Netzwerke. 
Spiele durchaus Lösungskonzepte für multilaterale Verhandlungs- und Koordinationsprobleme vorliegen, diese jedoch von einer mathematischen Komplexität sind, die sie für reale Anwendungsfälle unergiebig werden läßt.

Grundsätzlich lassen sich für Verhandlungsprozesse in Netzwerken drei Koordinationsprobleme, die auf unterschiedlichen Interdependenzbeziehungen basieren, unterscheiden (Thompson 1967, 54ff). Im ersten Fall geht es um die Verständigung über gemeinsame Standards (»coordination by standardization «) als Orientierungsgrundlage für individuelles Verhalten, in Fällen, in denen, in der Terminologie von Thompson, »pooled interdependence « zwischen den beteiligten Akteuren vorliegt. Als Beispiel könnten technische Normierungsverfahren, die Definition von Qualitåtsstandards oder auch von Arbeitssicherheitskriterien gelten. In diesen Fällen kann es durchaus Konflikte über die Wahl zwischen konkurrierenden Standards geben. Sind jedoch einmal verbindliche Standards, »routines or rules«, definiert und akzeptiert, können die Akteure ihre verbleibenden Optionen völlig autonom gestalten. In dieser Situation ist das Problem der großen Zahl nur bedingt relevant und relativ unkompliziert lösbar.

Im zweiten Fall geht es um »sequentielle Interdependenz«, zum Beispiel, wenn in Unternehmen oder auch Organisationen Prioritäten definiert oder Prozesse zeitlich aufeinander abgestimmt werden müssen. In solchen Situationen kann durch »coordination by plan « im Vorfeld der Umsetzung von Politiken oder Unternehmensstrategien die Komplexität reduziert werden. Auch in diesem Fall können die Akteure auf der Grundlage abgestimmter Sequenzen, »decision schedules«, autonom agieren. Das Problem der großen Zahl bleibt beherrschbar. In diesen beiden Fällen sind interaktive Koordinationsleistungen in einer Anfangsphase notwendig.

Im dritten Fall geht es um »reziproke Interdependenz«. Hier sind die Entscheidungsmöglichkeiten der beteiligten Akteure vom jeweiligen Verhalten der anderen abhängig, denn »the outputs of each become inputs for the others « (Thompson 1967, 55). Reziproke Interdependenzbeziehungen sind im Gegensatz zu den beiden bereits skizzierten Koordinationsmustern nur durch »coordination by mutual adjustment « möglich, stellen also hohe Anforderungen an die beteiligten Akteure. Dies gilt z. B. in oder zwischen Unternehmen, in denen die Aktivitäten zwischen Abteilungen - über Standardisierungen und Prioritätensetzungen hinaus - eng aufeinander abgestimmt werden müssen, aufeinander aufbauen und das gemeinsame Ergebnis von kontinuierlichen Abstimmungsprozessen abhängt. Ein weiteres Beispiel wären Politiknetzwerke, in denen die Steuerungsressourcen ( $z . \mathbb{B}$. Know how, Implementierungskapazitäten) über mehrere Akteure verteilt sind und Lösungen nur auf der Grundlage gemeinsamer Entscheidungsfin- 
dungsprozesse zum Erfolg führen können. In solchen Situationen verfügen Einzelakteure über Veto- und somit Blockadepotentiale (Scharpf 1993c, 140f). ${ }^{3}$ In diesen Fällen, die aufgrund der skizzierten gesellschaftlichen Entwicklungsdynamiken an Bedeutung ständig zunehmen, stellt sich das Problem der großen Zahl.

Aus dieser Problematik sind zwei wichtige Schlußfolgerungen zu ziehen: Erstens gibt es in einer zunehmenden Zahl von Politikfeldern und ebenso in der Ökonomie Herausforderungen, die gerade aufgrund ihrer Komplexität und der Verteilung von steuerungsrelevanten Ressourcen auf unterschiedliche Akteure hierarchisch-zentralistische Entscheidungsfindungsstrukturen vor unlösbare Aufgaben stellen. Interorganisatorische, horizontale Formen der Handlungskoordinierung verfügen in diesen Fällen über größere Problemlösungskapazitäten, denn

》... of course, the advantage of hierarchical coordination are lost in a world that is characterized by increasingly dense, extended and rapidly changing patterns of reciprocal interdependence, and by increasingly frequent, but ephemeral, interactions across all types of preestablished boundaries, intra- und interorganizational, intra- and intersectoral, intra- and international. Under such conditions, forms of horizontal self-coordination, whatever their comparative disadvantages, may be the only type that works ... (Scharpf 1993c, 141).

Um übermäßige Anforderungen an die Informationsverarbeitungs- und Konfliktregelungskapazität in Netzwerken zu verhindern, muß es ein Ziel sein, die Zahl der beteiligten Akteure, unter denen komplexe Aufgabeninterdependenz durch Verhandlungskoordination bewältigt werden kann, möglichst zu begrenzen. Dies gilt, wie gezeigt, insbesondere für den Fall »reziproker Interdependenz«.

Hieraus ergibt sich zweitens, daß Ansätzen mit Skepsis zu begegnen ist, die auf der Grundlage von Kritiken an einseitig zentralistischen Koordinationsund Entscheidungsstrukturen, eine möglichst weitgehende Dezentralisierung oder Arbeitsteilung in Unternehmen, Organisationen oder auch Regionen und Ländern als a priori effizienzsteigerndes Organisationsmodell anraten, um darauf aufbauend durch horizontale Handlungskoordinierung gemeinsame Problemlösungen anzusteuern. ${ }^{4}$ In Arbeiten mit dieser

Scharpf (1993c) verweist darauf, daß, wenn in solchen Fällen n Beteiligte zwischen s Optionen wählen können, die Identifikation eines Gesamtoptimums die Überprïfung von $s^{*}$ n Gesamtlösungen erfordert.

4 Viel rezipierte Arbeiten mit diesem Tenor finden sich z. B. in Teilen der Managementtheorie, in der die Diskussion über die »Abflachung von Hierarchien « nicht selten in naive Vorstellungen über die Leistungsfähigkeit großer Organisationen und Unternehmen einmünden, die mehr oder weniger vollständig auf Hierarchien verzichten und auf horizontale Handlungskoordination aufbauen (vgl. z. B. Peters 1987). In der Entwicklungspolitik wird von vielen Autoren und noch zahlreicheren Praktikern Dezentralisierıng und die Schwächung des Nationalstaates unkritisch als generelle Lösung für Entwicklungsblockaden empfohlen, ohne die Komplementarität zwischen zwischen nationalstaatlichen Aufgaben und Dezentralisierung zu thematisieren und zu berücksichtigen, daß 
Stoßrichtung wird oft (unter anderem) das trivale $\gg$ Problem der großen Zahl« außer acht gelassen. Wohlgemerkt sind hier Probleme angesprochenen, die sich insbesondere in Netzwerken, die durch »reziproke Interdependenz« gekennzeichnet sind, ergeben. Nichts spricht natürlich dagegen, z. B. zur Erhöhung des Informationsniveaus in privaten und öffentlichen Unternehmen, einem Politikfeld oder einem Wirtschaftssektor das Know how möglichst vieler Akteure zu poolen. Schwierigkeiten entstehen jedoch. wenn eine große Zahl von Akteuren, ein Gesamtergebnis erarbeiten müssen, das von den Entscheidungen und Handlungsweisen aller (oder vieler) abhängt. Diese Überlegungen schließen nicht aus, daß ein solches Vorgehen in Ausnahmesituationen als Strategie zur Entscheidungsfindung (z. B. zur Legitimierung einer Grundsatzentscheidung) sinnvoll sein kann.

\section{Die Zeitdimension von Entscheidungen}

Für jedes Organisations- und Steuerungsmuster stellt sich in der zeitlichen Dimension die entscheidende Frage, ob die institutionellen Bedingungen, in deren Rahmen der Entscheidungsprozeß abläuft, gewährleisten können, daß Politikentscheidungen nicht nur kurzfristigen Impulsen folgen, sondern auf die Entwicklung und Umsetzung dauerhaft tragfähiger Lösungen ausgerichtet werden können. Hinsichtlich dieser Problemstellung besitzen hierarchische Entscheidungssysteme rein theoretisch die größten Handlungsspielräume. Wenn sie unabhängig von anderen gesellschaftlichen Akteuren agieren können, sind sowohl an langfristigen Perspektiven und tragfähigen Problemlösungen orientierte Entscheidungen als auch kurzfristig ausgerichtete Politiken, die sich z. B. an Eigeninteressen der Entscheidenden orientieren, denkbar. Prinzipiell sind hierarchische Entscheidungssysteme jedoch in der Lage, Zukunfts- gegen Gegenwartsinteressen und strukturell wichtige Entscheidungen gegen vermachtete Partikularinteressen durchzusetzen. Zugleich sind hierarchische Entscheidungssysteme, die keinen Kontrollmechanismen unterliegen, freilich auch besonders anfällig

horizontale Selbstkoordination auf organisations- und handlungsfähige Akteure auf den entsprechenden gesellschaftlichen Ebenen angewiesen ist (vgl. z. B. Simon 1993, kritisch dazu Leffler 1994, kritische Anmerkungen am Beispiel der deutschen Entwicklungszusammenarbeit mit Bolivien macht Messner 1993, 45ff). Die Komplexität der Problematik berücksichtigend diskutiert Maggi (1994) das Verhältnis von Nationalstaat und Regionalisierung. In der ökonomischen Theorie wird zuweilen die Existenzberechtigung des Nationalstaates in Frage gestellt, da sich Weltwirtschaft und Weligesellschaft in eine mborderless world« auflöse, in der Unternehmen sich über Märkte ausłauschen und bestenfalls noch »regional states«, unterhalb der Ebene des Nationalstaates, politische Regulierungsfunktionen - im Sinne des Setzens von Rahmenbedingungen - wahrnehmen (vgl. z.B. affirmativ Ohmae 1993; im Sinne eines $»$ notwendigen Trends« marktwirtschaftlicher Entwicklung s. Knieper 1993). 
für opportunistisches Verhalten und »rent seeking-Strategien« der Entscheidenden (Ashoff 1988).

In majoritären Entscheidungssystemen kann aufgrund des Drucks durch Wahlen und unsichere Mehrheitsverhältnisse die Tendenz bestehen, kurzfristige Orientierungen zu präferieren und notwendige, aber in kurzer Frist "schmerzvolle " Entscheidungen in die Zukunft zu verschieben. In Netzwerken sind die Akteure zunächst von dem in majoritären Systemen bestimmenden Parteienwettbewerb und dem Druck, rasch Entscheidungen zu produzieren, die von außenstehenden Akteuren bzw. Wählern positiv gewürdigt werden, befreit. Die Akteure in den Netzwerken arbeiten vor allem an Lösungen für Probleme, die sie unmittelbar selbst angehen. Netzwerke sind ja gerade dadurch gekennzeichnet, daß die Rollen von Betroffenen und Entscheidern weitgehend zusammenfallen. ${ }^{5}$ Vor diesem Hintergrund besteht kein unmittelbarer Anreiz, kurzfristig orientierte Nutzenmaximierung zu betreiben.

Dennoch ergeben sich drei Problemkonstellationen. Erstens sind Situationen denkbar, in denen der individuelle Nutzen von an Netzwerken beteiligten Akteuren oder Organisationen kurzfristig kalkuliert wird, die Durchsetzung von »Systeminteressen « des Gesamtnetzwerkes dagegen eine längerfristige Orientierung verlangen. Dies kann z. B. der Fall sein, wenn die »Systemnutzen « sachlich auf einer anderen Ebene liegen, als die entscheidungsrelevanten individuellen Nutzen (Mayntz 1993, 48). Ein Beispiel: In einem regionalen technologie- und industriepolitischen Netzwerk, in dem Unternehmer(verbände), Ministerien, Gewerkschaften und Wissenschaftler zusammenwirken, um strukturpolitische Entscheidungen zur Stärkung der Wettbewerbsfähigkeit in einer Region vorzubereiten, könnte der »Systemnutzen « in einem radikalen, innovationsfördernden Strukturwandel bestehen. Zumindest ein Teil der Akteure, vor allem die, welche durch den Strukturwandel bedroht sind, werden in diesem Fall auf Domänesicherung ausgerichtet sein. Die Verfolgung individueller Nutzen stellt also in diesem Fall eine unsichere Basis für die Erarbeitung einer möglichst optimalen Problemlösung für das Gesamtsystem (in diesem Fall eine Wirtschaftsregion) dar. In das Verhandlungssystem ist demnach auch eine konservative, strukturerhaltende Tendenz eingebaut.

Zweitens dürften Akteure in Netzwerken Interesse an einer zeitlichen Stabilität der jeweiligen Verhandlungsstruktur haben, die, da es sich i. d. R. um freiwillige Formen der Koordination und Kooperation handelt, hergestellt werden muß (Scharpf 1993c, 60f). Das Interesse an dauerhafter,

Dies gilt natürlich nicht durchgängig. So können in Netzwerken auch Experten engagiert sein, die man nicht direkt als Betroffene bezeichnen kann. 
stabiler Kooperation ergibt sich aus der wechselseitigen Abhängigkeit von den Handlungsressourcen der anderen Beteiligten sowie den hohen Transaktionskosten, die durch den komplexen Aushandlungsprozeß entstehen. Kontinuierliche Zusammenarbeit ist Bedingung für die Erwartungssicherheit aller beteiligten Akteure. Aus dieser auf Stabilitätssicherung abzielenden Konstellation, entsteht ein Trend zu Konfliktvermeidung und inkrementellem Wandel. Innerhalb des Netzwerkes werden Diskussionen und Problemlösungen, die auch grundlegende Umorientierungen miteinschlieBen würden und die die Interessen wichtiger Akteure des Netzwerkes negativ tangieren könnten, eher zurückgestellt. Status-quo-Orientierungen, die auf in der Vergangenheit abgeschlossenen Vereinbarungen basieren und weniger die Gegenwarts- geschweige denn die Zukunftsinteressen reflektieren, sind denkbar.

Gelingt die Stabilisierung des Netzwerkes, so kann drittens das dann erreichte hohe Maß an sozialer Kohäsion Problemlösungen begünstigen, die eher einer »parametrischen « als einer »strategischen Rationalität« entsprechen. Unter »parametrischer Rationalität« wäre eine starke Präferenz für Entscheidungen zu verstehen, die sich eng entlang des traditionellen Entwicklungspfades bewegen (Grabher 1994, 79). Die Herausbildung einer »Konsens-Kultur« (Grabher 1994, 80) und symbiotischer Beziehungen zwischen den Netzwerkakteuren, die inkrementellen Wandel durchaus begünstigen, können zu »kollektivem Konservatismus (Kuran 1988) führen und notwendige Strukturveränderungen verlangsamen und blockieren.

Netzwerke sind daher vermutlich leistungsfähig, wenn es um Strukturgestaltung innerhalb eines etablierten und dynamischen Entwicklungspfades geht. Hier dürften sich im wesentlichen Positivsummenspiele ergeben. Netzwerke sind jedoch aufgrund der beschriebenen Trends zu konservativen, strukturerhaltenen Entscheidungen und inkrementellem Wandel vor größere Schwierigkeiten gestellt, wenn strukturelle Krisen zu bewältigen sind und Umbrüche eingeleitet und gesteuert werden müssen. Man könnte mit anderen Worten auch sagen, daß Netzwerke in Politikfeldern, die durch die wechselseitige Abhängigkeit der Akteure von den Steuerungsressourcen anderer gekennzeichnet sind (z. B. Technologie- und Industriepolitik) hohe Leistungsfähigkeit aufweisen, wenn auf der Grundlage etablierter Gegenwartsinteressen und eines stabilen Erwartungshorizontes Strukturgestaltung voranzutreiben ist. Netzwerke sind weniger flexibel und vor größere Herausforderungen gestellt, wenn Erwartungshorizonte weitgehend modifiziert werden müssen und es um die Durchsetzung von Zukunfts- gegen etablierte Gegenwartsinteressen geht. 


\section{Institutionelle Konsolidierung von Netwwerken: Bedingung und Problem fiir deren Funktionsfähigkeit}

Die wechselseitige Abhängigkeit der in einem Netzwerk agierenden Akteure impliziert also einen Trend zur Verstetigung der Akteursbeziehungen und somit ein Mindestmaß an institutioneller Konsolidierung - in den hier relevanten Fällen unterhalb der Schwelle der Konstituierung einer formalen Organisation, wodurch die Netzwerkstruktur selbst aufgegeben würde. ${ }^{6}$ Relativ stabile Kooperationsbeziehungen, die durch ein spezifisches $\mathrm{Mu}$ ster gegenseitig akzeptierter organisatorischer Identitäten, Kompetenzen und Interessensphären, also einen minimalen institutionellen Grundkonsens, charakterisiert sind, erleichtern es, Interessenkonflikte zwischen den Netzwerkakteuren durch Kompromisse zu lösen und die rücksichtslose Nutzenmaximierung auf Kosten einiger Akteure wenn nicht vollständig auszuschließen, so doch zu reduzieren (Mayntz 1993, 49f).

Der Druck, das Netzwerk zu stabilisieren und Kompromisse zu finden, steigt mit der Abhängigkeit von den Steuerungsressourcen anderer Netzwerkakteure sowie der Dauer der Kooperationsbeziehungen, die zu entsprechenden Transaktionskosten führen und daher eine Aufgabe der Netzwerk-Kooperation unattraktiv machen. Da die Entstehung von Netzwerken in der Regel auf wechselseitige Interdependenzbeziehungen zwischen den Akteuren zurückgeht, sind die die »costs of exit « (Scharpf 1991b, 288) generell hoch und sie steigen stetig an, weil sich im Verlauf der Netzwerkkooperation beachtliche Transaktionskosten kumulieren.

Die Konsolidierung und Verstetigung der Beziehungen von Netzwerkakteuren sind demnach in der Regel eine wesentliche Bedingung für die Funktionsfähigkeit von Netzwerken. Gelingt die Stabilisierung nicht, sind lose gekoppelte Netzwerke von Desintegration bedroht. Der Übergang von den »weak ties«, die die prekären Beziehungsgeflechte entstehender Netzwerke prägen, zu »stronger ties« kann jedoch zugleich potentielle Schwächen von Netzwerkstrukturen zu Tage fördern. Netzwerke agieren im Spannungsfeld von Desintegration, Anpassungsgefahr und »endless disagreement« zwischen den beteiligten Akteuren auf der einen Seite sowie funktionaler und kognitiver Blockierung durch ein zu hohes Maß an sozialer Kohäsion auf der anderen Seite. In diesem Spannungsfeld lassen sich vier Problemdimensionen ausmachen.

Die potentiell retardierende Funktion der Kompromißlogik: Zunächst einmal implizieren interdependente und enger werdende Beziehungsstruktu--

Natürlich gibt es auch eine Vielzahl von Fällen, in denen aus Netzwerken neue formale Organisationen oder Unternehmen evolvieren. Dies gilt z. B., wenn Unternehmen ihre Zulieferer in das eigene Unternehmen integrieren. 
ren in Netzwerken und die Notwendigkeit, Kompromisse anzusteuern, das Problem, daß die Neu- oder Umverteilung von Macht und anderen Ressourcen (z. B. Geld, Zugang zu Märkten oder Informationen, sozialer Status) zwischen den Akteuren, die zur Erreichung eines Zieles für das gesamte Netzwerk wichtig sein können, gegen deren Willen schwierig sind. Während in hierarchisch strukturierten Entscheidungssystemen die Durchsetzung von Interessen der Zentralgewalt unproblematisch ist und in Mehrheitssystemen die Möglichkeit (und auch Gefahr) der Durchsetzung von Interessen gegen Minderheiten existiert, sind Netzwerke in der Regel auf einvernehmliche Entscheidungen angewiesen. In dieser Konstellation ist freilich eine starke konservative Tendenz eingebaut. Etablierte Interessen können sich gegen Zukunftsinteressen durchsetzen:

»... unanimous decisions permit each party to defend the existing pattern of distribution, while majoritarian and hierarchical decision rules create the possibility of involuntary redistribution...«(Scharpf 1991a, 60).

Funktionale Blockierungen: Da die Kosten für das Scheitern von Verhandlungen oft hoch sind (hohe »exit costs «, Gefahr von »endless disagreement $\ll$ ), ist es wahrscheinlich, daß zur Konfliktvermeidung Lösungen, die die Interessen etablierter und strategisch wichtiger Akteure negativ tangieren, unterbleiben und Strategien innerhalb des existierenden Entwicklungspfades angesteuert werden. Scharpf hat für diese Konstellation den Begriff »joint-decision trap« geprägt. Netzwerke

wwith high internal consensus requirements will find it difficult to exploit new opportunities that are attractive on balance, but not for each member individually, and they will encounter similar difficulties when it would be rational to cut their losses in response to worsening conditions. « (Scharpf 1991b, 285)

Kognitive Blockierungen: Diese funktionalen Innovationshemmnisse können durch kognitive Blockierungen überlagert werden. Langfristig stabile persönliche Beziehungen zwischen Netzwerkakteuren begünstigen die Herausbildung gemeinsamer Orientierungen, Sichtweisen, Verhandlungsstile oder auch Vorurteile. Die Kehrseite sozialer Kohäsion als produktiver Ressource für das Funktionieren von Netzwerken sind kognitive Verengungen durch überzogene Vereinheitlichungen von Sichtweisen und zu symbiotische Beziehungen zwischen Netzwerkakteuren. Alternativen, neue Entwicklungswege, Lösungsansätze und Strategien jenseits eingefahrener Optionen werden nicht zur Kenntnis genommen. Das Denken und Handeln im Netzwerk droht ïberzogen pfadabhängig, strukturkonservativ und borniert zu werden. Wichtig ist es zu betonen, daß funktionale und kognitive Blockierungen in Netzwerken als Problem insbesondere dann auftauchen, wenn eingeschlagene Entwicklungspfade verlassen werden müssen, also inkrementelle soziale, politische und ökonomische Lösungen zur Überwin- 
dung eines Problemkomplexes, für deren Erarbeitung das Netzwerk aufgrund seiner interaktiven, Know-how bündelnden und strukturgestaltenden Funktionen besonders geeignet ist, nicht mehr ausreichen.

Grabher nennt als Beispiel funktionale und kognitive Blockierungen in traditionellen Industrieregionen (wie z. B. dem Ruhrgebiet), in denen ehemals durchaus produktive Netzwerke trotz langandauernder Krise »statt ein neues Heilmittel auszuprobieren, ... mit der zunehmenden Verschlimmerung des Leidens lediglich die Dosis des alten erhöht (wurde) (Grabher 1994, 79). Andere prominente Beispiele für den »high degree of inertia (Scharpf 1991b, 285) aufgrund funktionaler und kognitiver Blockierungen wären, um weitere Beispiele aus der Bundesrepublik anzuführen, der starke Widerstand von Teilen des Gesundheitssystems gegen eine Serie von Reformversuchen oder auch die Reformunfähigkeit im Hochschulbereich. Die institutionelle Konsolidierung von Netzwerken und die Logik des Kompromisses sind also Bedingungen dafür, in kollektiven Verhandlungssystemen Entscheidungsblockaden zu verhindern, Interessensausgleich sicherzustellen und Desintegrationstrends zu reduzieren, jedoch »kein ungetrübter Segen« (Mayntz 1993, 50).

Ingroup-outgroup Logik (»Luhmann-Falle«): Die Stabilisierung von Netzwerken nach Innen geht einher mit der Herausbildung von gemeinsamen Regeln, Konventionen und Routinehandlungen. Erst in einem solchen Handlungskontext kann sich zwischen den Akteuren Vertrauen, Reziprozität und die Fähigkeit, die Verhaltensweisen der anderen Akteure zu antizipieren, herausbilden. Die Entstehung solcher »ingroup-Strukturen« basiert auf der Konstituierung von »ingroup-outgroup boundaries«. Eine Vielzahl von Untersuchungen zeigen, daß $»$ trust among insiders, ..., could arise from common distrust of outsiders. « (Scharpf 1991b, 296)

Renate Mayntz und Friedhelm Neidhardt haben diesen Mechanismus am Beispiel der bundesdeutschen Parlamentarier untersucht. Sie stießen auf starke Solidaritätsnormen innerhalb der politischen Fraktionen, ritualisierte »Feindschaften zwischen den Fraktionen von Regierung und Opposition sowie typisches »ingroup Verhalten« der Parlamentarier als Gesamtgruppe, z. B. zur Vertretung ihre Interessen als »politische Klasse « gegenüber den Medien (Mayntz/Neidhardt 1989). Scharpf verweist auf eine Untersuchung von Edward Banfield, der in süditalienischen Dörfern und Städten Beziehungstrukturen entdeckte, die er als »amoral familism« bezeichnete: »trust and cooperation within the family coexisted with complete distrust and morally unrestrained cheating among individuals not belonging to the same family (Scharpf 1991b, 296). Grabher verweist darauf, daß auch in einigen der für ihre Leistungsfähigkeit inzwischen berühmten »industrial districts« Norditaliens, deren Wettbewerbsfähigkeit inbesondere auf dichte 
Netzwerkstrukturen in den Industrieregionen zurückgeführt werden kann, zugleich Tendenzen in Richtung auf »hermetische Abgeschlossenheit zu beobachten sind (Grabher 1994, 93ff).

Allgemeiner formuliert scheint eine gewisse »feindliche Orientierung « gegeniuber der Umwelt die Konsolidierung von ingroups, also in unserem Falle von Netzwerken, und die gruppeninterne Entstehung von Vertrauensbeziegungen zu begünstigen. Weitere Beispiele wären Beziehungsmuster zwischen Innen- und Außenwelt des organisierten Verbrechens, in der internationalen Politik, zwischen Sportteams, Unternehmensgruppen oder auch Netzwerken in der Wissenschaft. Schon Max Weber thematisierte diese Phänomene unter dem Stichwort »Soziale Schließung«. ${ }^{7}$

Die Herausbildung von funktionstiichtigen Netzwerken auf der Grundlage von »ingroup-outgroup boundaries« kann zu Segmentierungstendenzen zwischen sich voneinander abschottenden, nicht kommunizierenden Subsystemen führen. Eine solche Tendenz ist in hoch interdependenten Gesellschaften außerordentlich gefährlich. Sie begünstigt zwei divergierende Typen von Externalisierung:

- erstens, aktive Strategien von einseitig ingroup-orientierten und ihrer Umwelt tendenziell »feindlich « gegenüberstehenden Netzwerken (»boundaries of distrust « (Scharpf 1991b, 297)), Kosten und negative Externalitäten bewußt auf Dritte zu verlagern (»opportunism with guile $\ll) ;{ }^{8}$

- zweitens, Aktivitäten von borniert agierenden Netzwerken, die aufgrund fehlender Abstimmung mit anderen Subsystemen bzw. Netzwerken zu unkontrollierbaren, weil nicht berücksichtigten Handlungsfolgen führen (nicht-intendierten Effekte). Dieser zweite Typ von Externalisierung dürfte häufiger auftreten als der erste, da das Verhältnis zwischen den durch den

7 Weber $(1960,35)$ schreibt: »Eine soziale Beziehung (gleichviel ob Vergemeinschaftung oder Vergesellschaftung) soll nach außen offen heißen, wenn und insoweit die Teilnahme an dem an ihrem Sinngehalt orientierten gegenseitigen sozialen Handeln, welches sie konstituiert, nach ihren geltenden Ordnungen niemand verwehrt wird, der dazu tatsächlich in der Lage und geneigt ist. Dagegen nach außen geschlossen dann, insoweit und in dem Grade, als ihr Sinngehalt oder ihre geltenden Ordnungen die Teilnahme ausschließen oder beschränken oder an Bedingungen knüpfen. Offenheit und Geschlossenheit können traditionell oder affektuell oder wert- oder zweckrational bedingt sein. Die rationale Schließung erfolgt insbesondere durch folgenden Sachverhalt: Eine soziale Beziehung kann den Beteiligten Chancen der Befriedigung innerer oder äußerer Interessen eröffnen, sei es dem Zweck oder dem Erfolg nach, sei es durch solidarisches Handelin oder durch Interessenausgleich. Wenn die Beteiligten von ihrer Propagierung eine Verbesserung ihrer eigenen Chancen nach Maß, Art, Sicherung oder Wert erwarten, so sind sie an Offenheit, wenn umgekehrt von deren Monopolisierung, so sind sie an Schließung nach außen interessiert.« (Hervorhebungen im Orginal)

8 Beispiele wären Unternehmensstrategien zur Externalisierung ökologischer Kosten zu Lasten der Umwelt, Strategien der Bundesregierung zur Verschiebung von Sozialkosten auf regionale und lokale Ebenen, Müllexporte in die Dritte Welt. 
ingroup-Mechanismus stabilisierten Netzwerken und ihrer Umwelt i. d. R nicht so sehr durch Antagonismus (im Sinne von »opportunism with guile $\ll)$, sondern durch Indifferenz, Gleichgültigkeit der Handelnden gegenüber negativen Externalitäten, die bei der Verfolgung eigener Interessen verursacht werden, geprägt sind (»boundaries of irrelevance« (Scharpf 1991b, 298)).

Beide Typen von Externalisierung basieren darauf, daß die ingroup-Mechanismen zur Stabilisierung der Netzwerke »may segregate systems of communications, but they do not have the power to interrupt chains of real interdependence among functional subsystems (Scharpf 1991a, 299).

Die herausgearbeitete Externalisierungs- und Segmentierungsproblematik entspricht der These Luhmanns von der zunehmenden Selbstreferentialität von Subsystemen in modernen Gesellschaften und der Unmöglichkeit »intersystemischer Diskurse«. Diese »Luhmann-Falle« wird demnach, wie Lindenberg zu Recht betont, nicht nur durch »opportunism with guile « zwischen Akteuren bzw. Akteursgruppen ausgelöst, sondern eben auch durch »strong solidarity among insiders «, die auf Mißtrauen gegenüber outsiders basieren kann (Lindenberg 1988, 43).

Zugleich muß festgehalten werden, daß die »Luhmann-Falle « eine reale Möglichkeit, nicht jedoch die generelle Regel darstellt. Unter der Bedingung generalisierten Mißtrauens zwischen Akteuren unterschiedlicher Netzwerke wären moderne und sich modernisierende Gesellschaften nicht funktions- und überlebensfähig. Wenn funktionale Differenzierung, als eine der wesentlichen Charakteristika moderner Gesellschaften, in strukturelle und generalisierte Segmentierung zwischen Subsystemen umschlägt, droht soziale Desintegration. Neben der »ingroup-outgroup-Logik « sowie der Möglichkeit der gesellschaftlichen Akteursgruppen und Netzwerke des »playing side-by-side according to their own specific logic (Scharpf 1991 b, 298), als Produktivitäts- und Kreativitätsressource ausdifferenzierter Gesellschaften, existieren offenbar Mechanismen, die, über die entstehenden und notwendigen Abgrenzungen zwischen Netzwerken und Subsystemen hinaus, intersysternische Kommunikation ermöglichen und damit den realen Interdependenzen Rechnung tragen. Scharpf spricht von »mechanisms that are able to connect as well as to separate « (Scharpf 1991b, 299) ${ }^{9}$, um diéses Spannungsfeld zu charakterisieren.

9 Scharpf (1991b, 299) führt dazu aus: »In the real world, of course, such interactions must occur continously - between scientists and corporate managers in industrial research, between lawyers and politicians in the legislative process, and across all other boundaries as well.« 


\section{Das Koordinationsproblem}

»Oh, what a tangled web we weave ..." Sir Walter Scott, zitiert nach: Shubik (1987), S. 33.

Netzwerktheoretiker unterstellen häufig a priori eine höhere Rationalität von Netzwerkkoordination im Vergleich zu hierarchisch oder marktförmig verfaßten Systemen der Entscheidungsfindung. Zuweilen wird dies mit Verweis auf das berühmte Coase-Theorem begründet, das besagt, daß alle Wohlfahrtsgewinne, die durch ideale hierarchische Koordination erreichbar wären, auch durch freiwillige Vereinbarungen zwischen autonomen, ausschließlich egoistisch-rationalen Akteuren realisiert werden können (Coase 1960). ${ }^{10}$ Bereits die herausgearbeiteten Problemdimensionen verdeutlichen, daß eine derart pauschale Aussage über die Möglichkeiten des Netzwerkversagens leicht hinwegtäuschen kann. Scharpf hat darüberhinaus nachgewiesen, daß nicht nur Markt- und Hierarchie, sondern auch freiwillige Verhandlungslösungen in Netzwerken spezifische Koordinationsprobleme aufwerfen und die Voraussetzungen für das Funktionieren von Koordination in netzwerkartigen Verhandlungssystemen außerordentlich komplex sind. ${ }^{11}$

Als Indikatoren zur Messung der Effizienz von Koordinationsleistungen greift Scharpf auf das Pareto-Optimum sowie das Kaldor-Kriterium zurück. Das Pareto-Optimum, als dem wohlfahrtstheoretischen Maßstab der Ökonomie, definiert bekanntlich die Allokation knapper Ressourcen auf konkurrierende Verwendungsmöglichkeiten dann als effizient, wenn keine Änderungen mehr denkbar sind, die den Nutzen mindestens eines Beteiligten erhöhen würde, ohne daß irgend ein anderer deshalb schlechter gestellt werden müßte. Dieser wohlfahrtstheoretische Indikator vernachlässigt bewußt Aspekte wie Verteilungsgerechtigkeit oder die Möglichkeit der Umverteilung von Besitzständen. Einen politisch interessanteren Wohlfahrtsmaßstab bietet das Kaldor-Kriterium, das alle Maßnahmen positiv bewertet, deren Nutzen für die Begünstigten groß genug ist, um daraus alle durch die Maßnahme Benachteiligten voll entschädigen zu können (Kaldor 1939).

Auf den ersten Blick erscheint der dem Gemeinwohl verpflichtete nach innen und außen souveräne, demokratische Nationalstaat, also eine hierarchische Entscheidungsinstanz, am ehesten geeignet um dem anspruchsvollen

10 Oft werden die von Coase betonten Voraussetzungen für die Funktionsfähigkeit seines Theorems nicht genannt: erstens werden Transaktionskosten außer acht gelassen und zweitens werden Verteilungsfragen ignoriert.

11 Die folgenden Ausführungen basieren im wesentichen auf Scharpf (1991); vgl. auch Scharpf (1992b und 1993a). 
Kaldor-Kriterium Rechnung zu tragen. Dieser kann sich über den Einspruch einzelner Betroffener hinwegsetzen und ist theoretisch in der Lage, Gemeinwohlmaximierung zu betreiben. Auf den zweiten Blick werden die Grenzen des hierarchischen Staatskonzeptes deutlich: der Staat hat an Souveränität nach innen und außen verloren, die Gemeinwohlorientierung des Staates kann nicht einfach voluntaristisch vorausgesetzt werden, und der Staat ist in vielen Politikfeldern auf die Kooperation mit anderen gesellschaftlichen Akteursgruppen angewiesen.

Wie steht es nun um die Koordinations- und Entscheidungsfähigkeit in Netzwerken unter Berücksichtigung ihrer wohlfahrtstheoretischen Effekte. Scharpf exemplifiziert zunächst die Koordinationsprobleme von zwei Akteuren (s. Abbildung). Er setzt zwei egoistisch rationale Akteure ( $X$ und Y) mit voneinander unabhängigen Nutzenvektoren und eine Reihe von Handlungsoptionen (A; B; C; D; E) voraus. Der status quo wird durch den Ursprung des Koordinatensystems gekennzeichnet. Unterstellt man nun, daß die beiden Akteure ihre Handlungsoptionen durch einseitige Entscheidungen realisieren können, dann werden für $(X)$ nur Vorhaben, die rechts von der $\mathrm{Y}$-Achse liegen und für (Y) nur Vorhaben, die oberhalb der $\mathrm{X}$ Achse liegen, akzeptabel sein. Demnach würde (X) die Projekte (D) und $(\mathrm{E})$ ignorieren, und die Präferenz von $(\mathrm{X})$ läge bei $(\mathrm{A})$; für $(\mathrm{Y})$ wären $(\mathrm{A})$ und $(E)$ inakzeptabel und (D) die präferierte Lösung.

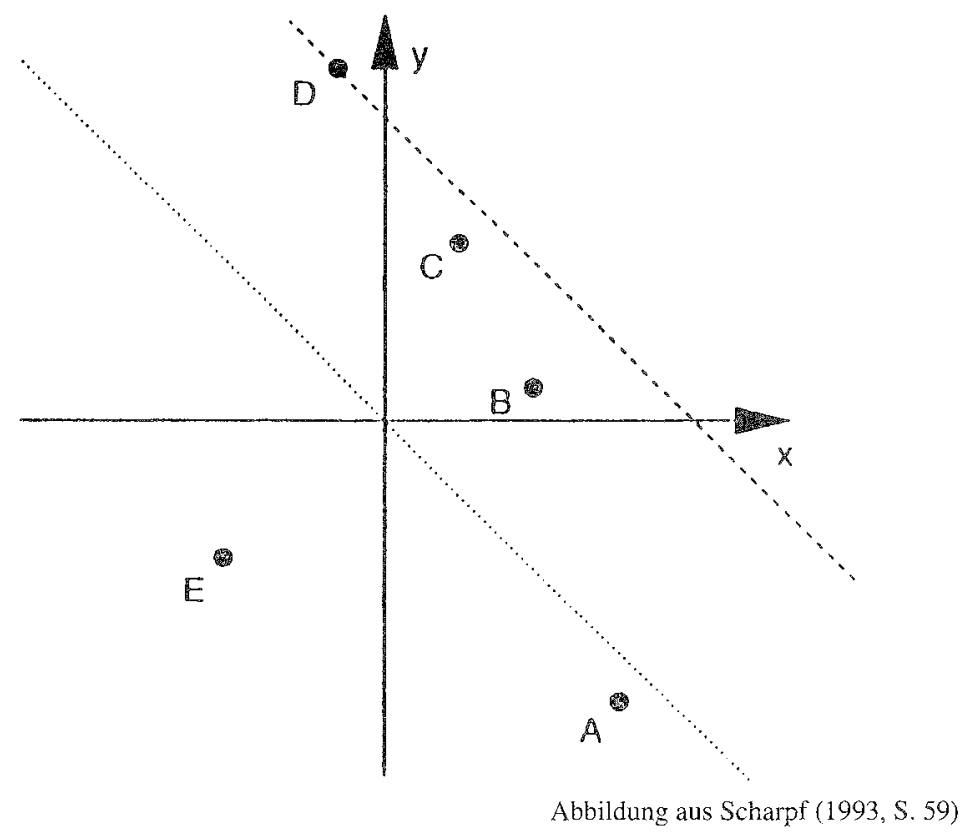


Eine wohlfahrtstheoretische Bewertung dieser einseitig präferierten Lösungen unter dem Pareto-Kriterium kommt zu dem Ergebnis, daß beide nicht akzeptabel sind, weil bei jeder von ihnen eine Verbesserung des status quo für die eine Seite einhergeht mit einer Verschlechterung für die andere Seite. Zu einem anderen Ergebnis kommt man durch die Anwendung des Kaldor-Indikators. Der Unterschied wird leicht deutlich, wenn beide Projekte - also (A) und (D) - aus der Perspektive eines hierarchischen Koordinators beurteilt werden - z. B. einer Unternehmenszentrale mit zwei Abteilungen. Auf der Grundlage seiner Nutzenfunktion $(U=U x+U y)$ wäre offensichtlich kein Projekt unterhalb der Nordwest-Südost-Diagonalen überhaupt akzeptabel. Vorhaben würden umso attraktiver, je weiter nordöstlich sie von dieser Diagonalen angesiedelt wären. Dementsprechend würde der hierarchische Koordinator das von (X) präferiert Projekt (A) verhindern, das von (Y) unterstiitzte Projekt (D) jedoch billigen, obwohl dadurch die Interessen von $(X)$ verletzt werden, da der Gesamtnutzen höher ist als die Verluste von $(\mathrm{X})$.

Was würde passieren, wenn die Koordination statt durch die Hierarchie durch Verhandlungen zwischen $(\mathrm{X})$ und $(\mathrm{Y})$ zustande kommen müßte? Gehen wir von einem Zwangsverhandlungssystem aus (in dem also die bereits diskutierten einseitigen Aktionen ausgeschlossen sind) ${ }^{12}$, in dem es um eindeutig fixierbare Vorhaben geht, bei denen Ausgleichzahlungen zunächst ausgeschlossen sind. Unter diesen Voraussetzungen würde $(\mathrm{X})$ alle Lösungen ablehnen, die links von der (Y)-Achse liegen und $(Y)$ diejenigen, die unterhalb der $(X)$-Achse angesiedelt sind. Für eine Einigung kämen demnach überhaupt nur pareto-superiore Optionen im nordöstlichen Quadranten in Frage. Unter diesen Lösungsmöglichkeiten würde (X) das Projekt (B) und (Y) das Vorhaben (C) präferieren. Für keinen von beiden gäbe es einsichtige Gründe, den Wünschen des anderen nachzugeben. Der Versuch einer Koordination durch Verhandlungen stößt also auf drei spezifische Probleme:

- Lösungen, die die aggregierte Wohlfahrt maximieren, werden systematisch ignoriert, wenn sie nicht gleichzeitig auch für jeden der Netzwerkakteure eine Verbesserung gegenüber dem status quo implizieren (also nicht in dem nord-östlichen Quadranten liegen).

12 Zwangsverhandlungsysteme können grundsätzlich aus zwei unterschiedlichen Gründen enstehen. Entweder dadurch, daß wichtige Steuerungsressourcen auf unterschiedliche Netzwerkakteure verteilt sind und daher eine wechselseitige Ahhängigkeit entsteht, die einseitige Aktionen nur um den Preis der Blockade des Netzwerkes und damit die Handlungsunfähigkeit aller Beteiligten zuläßt, oder dadurch, daß der Staat gesellschaftliche Akteursgruppen unter Androhung von Sanktionen dazu zwingt, gemeinsame Problemlösungen anzusteuern. 
- Für den Fall, daß unter diesen Bedingungen mehr als eine pareto-superiore Lösung möglich sein sollte, besteht die Gefahr, daß der Streit über die Wahl der Lösungen, die Verhandlungen blockiert.

- Daraus folgt, daß Netzwerke nur dann in der Lage sind, das Kaldor-Optimum anzusteuern, wenn sie sich nicht von kompetitiven oder gar feindlichen Handlungsorientierungen gegenüber anderen Netzwerkteilnehmern (im Sinne von »dem anderen Schaden zufügen «) leiten lassen.

Diese Problemdimensionen verschärfen sich mit zunehmender Zahl von Beteiligten, da es dann zu einer Kumulation von Veto-Positionen kommen kann (vgl. die Ausführungen zum »Problem der großen Zahl«).

Modifiziert man die bisherigen Voraussetzungen dahingehend, daß Ausgleichszahlungen, mit denen die Gewinner und Verlierer entschädigt werden können, möglich sind und als Instrument eingesetzt werden, so sind unter Vernachlässigung der Transaktionskosten - Lösungen für die skizzierte Problematik denkbar. Im Falle freiwilliger Verhandlungen in Netzwerken, müßte der Akteur, der von einem Projekt Nachteile zu befürchten hätte, die daran interessierten für deren Verzicht auf das Projekt bezahlen. In einem Zwangsverhandlungsystem (z. B. wenn Eigentumsrechte gegen negative Externalitäten rechtlich geschützt sind) müßten die Ausgleichszahlungen in die entgegengesetzte Richtung fließen.

In beiden Fällen würden die Verhandlungen zwischen den egoistisch-rationalen Akteuren die aggregierte Wohlfahrt der Beteiligten maximieren. Um dies am Beispiel der Abbildung zu erläutern: (Y) hätte durch die Umsetzung des Projektes (A) mehr zu verlieren, als (X) dabei gewinnen könnte. Er würde demnach in einem freiwilligen Verhandlungssystem dieses Vorhaben durch Ausgleichszahlungen verhindern. Umgekehrt hätte (Y) mit Projekt (D) mehr zu gewinnen als $(X)$ verlieren würde; er würde deshalb in einem Zwangsverhandlungssystem (X) dessen potentielles Veto abkaufen. Unter beiden Bedingungen (und unter Vernachlässigung von Transaktionskosten) würden demnach nur Projekte oberhalb der Diagonalen realisiert und die Akteure würden sich auf Lösungen einigen, die auf der am weitesten nordöstlich gelegenen Isoquante lokalisiert sind und demgemäß die aggregierte Wohlfahrt der Beteiligten maximieren.

Die Einbeziehung von zwei weiteren Faktoren erschwert jedoch die Koordinationsprobleme. Erstens erhöht, wie bereits angesprochen, eine steigende Zahl von Beteiligten die Transaktionskosten. Gravierender ist aber zweitens, daß die bisherigen Überlegungen auf der Verfügbarkeit von eindeutig fixierbaren Lösungen und der Möglichkeit monetärer Ausgleichszahlungen basierten. Beides sind plausible Annahmen in ökonomischen Kontexten, in denen Geld als allgemeines Äquivalent fungiert. In diesen Fällen können Ausgleichszahlungen oder Schattenpreise die Koordination 
zwischen Unternehmen, oder semiautonomen Abteilungen innerhalb eines Unternehmens oder in manchen Fällen auch im öffentlichen Bereich oder zwischen öffentlichen und privaten Akteuren ermöglichen. In vielen politischen Konstellationen läßt sich jedoch die Bewertung von Lösungen kaum in Geldwerten ausdrücken. ${ }^{13}$ Ausgleichszahlungen können zudem auch unter moralischen Gesichtspunkten unakzeptabel sein; man denke nur an Phänomene wie Kinder- oder Organhandel.

Scharpf verweist darauf, daß auch unter diesen Bedingungen Annäherungen an die Wohlfahrtseffekte des Coase-Theorems denkbar sind, wenn separate Projekte mit komplementären Kosten-Nutzen-Bilanzen zu Verhandlungspaketen gebündelt werden können, durch die eine im ganzen ausgeglichene Interessenbilanz erreicht werden kann. Arbeiten von Benz und Zintl zu diesem Problemfeld zeigen am Beispiel der Koordination zwischen den deutschen Bundesländern, daß das Hauptproblem in diesen Fällen darin besteht, ein gemeinsames Verständnis von Interessenausgleich und Verteilungsgerechtigkeit herauszubilden (Benz 1992; Zintl 1991). Die Funktionsfähigkeit von Netzwerken ist demnach stark von den Handlungsorientierungen der Akteure, der Fähigkeit der Definition von eigenen, konfliktiven und gemeinsamen Interessen und der Fähigkeit zum Kompromiß abhängig. Nur so können die skizzierten Verhandlungsblockaden aufgebrochen werden.

Da Fälle immer häufiger werden, in denen in Netzwerken diese nicht oder nur schwer monetarisierbaren Gewinne und Verluste zu verteilen sind, zugleich jedoch hierarchische Mechanismen der Entscheidungsfindung, die qua »Diktat « in der Lage wären, diese Problematik zu lösen, aus den oben diskutierten Gründen in vielen Politikbereichen überfordert sind, ist es nicht verwunderlich, daß in den letzten Jahren Theorien der Verteilungsgerechtigkeit einen ungeahnten Aufschwung genommen haben. ${ }^{14}$ In den 70er Jahren schien diese Frage, zumindest in den westeuropäischen Ländern während der Blütephase des Wohlfahrtsstaates obsolet zu sein. Fragen der Gerechtigkeit sowie die Umsetzung von Politiken zur Sicherung und Ver-

13 Vgl. z. B. in Deutschland die »Netzwerkverhandlungen« über einen »Energiekonsens«; generell Verhandlungen innerhalb der Gesellschaft iber Unweltprobleme, deren Kosten oft kaum quantifizierbar sind und zudem Zukunftsinteressen betreffen, für die es noch keine Interessenvertreter gibt.

14 Dabei steht in der Regel die Gerechtigkeitstheorie von John Rawls (1971 und 1993) im Mittelpunkt. In der aktuellen Diskussion - vgl. z. B. Dahrendorf (1992), Tugendhat (1993) und Honneth (1994) - um Gerechtigkeit bestimmen sich die Positionen der jeweiligen Theoretiker - von den radikalliberalen Positionen Nozicks (1974) bis zu dem konservativen Flügel der Kommunitaristen (vgl. als Überblick Honneth 1993) häufig durch ihre Stellung zu Rawls. Zur Frage der sozialen Gerechtigkeit im weltgesellschaftlichen und weltwirtschaftlichen Kontext und mit Bezug auf Rawls und die Kommunitarismusdebatte vgl. insbesondere Mármora (1992/1994). 
besserung von Lebenschancen und Verteilungsgerechtigkeit wurden an den Staat delegiert. In den 80er Jahren, der Dekade der Hegemonie des Neoliberalismus, schien die naive (und letztlich unsoziale) Illusion der Monetarisierbarkeit aller (oder fast aller) Beziehungen zwischen Menschen umsetzbar und vor allem wünschbar. Die Frage sozialer Gerechtigkeit wurde privatisiert und zu einem Problem der $\gg$ Leistungsfähigkeit des Individuums «. Die neoliberale Theorie beschäftigte sich mit der Frage der Verteilungsgerechtigkeit nur im Sinne von Allokationseffizienz im Rahmen des Pareto-Optimums; wohlfahrtstheoretische Überlegungen in die Richtung von Kaldor wurden strikt abgelehnt (Selten 1986). In den 90er Jahren zeichnet sich aufgrund der zunehmenden Bedeutung von Netzwerken zur Steuerung von Wirtschaft und Gesellschaft ein erneuter Wechsel ab. Die gesellschaftlichen Akteure und Gruppen sind gezwungen, zur Herstellung der Funktionsfähigkeit ihrer Netzwerkbeziehungen, gemeinsame Bewertungs- und Gerechtigkeitsvorstellungen zu entwickeln. Diese Aufgaben, die zuvor dem Staat und dann den Individuen zugeordnet wurden, müssen nun in interaktiven Kommunikationsprozessen abgearbeitet werden. Die Bewältigung dieser neuen Anforderungen ist besonders schwierig und in ihren gesamtgeschaftlichen Konsequenzen ambivalent, da netzwerkspezifische Gerechtigkeits- und Verteilungsvorstellungen entstehen können, die gesellschaftliche Segmentierungstendenzen noch verstärken. Dieser Fall tritt ein, wenn die Gerechtigkeitsregeln des einen Netzwerkes nicht mit denen der anderen, mit denen möglicherweise reale Interdependenzen existieren, kompatibel sind.

\section{Das Verhandiungsdilemma}

Die Auseinandersetzung mit den Koordinationsproblemen hat verdeutlicht, daß die »motivationalen Anforderungen (Honneth 1994, 12) ${ }^{15}$ für die Leistungsfähigkeit von Netzwerken bedeutend sind. Es wurde herausgearbeitet, daß die Koordination in Netzwerken und die Maximierung des aggregierten Nutzens im Regelfall nur dann gelingt, wenn die Akteure in der Lage sind, sich über die Verteilung der anfallenden Kosten und Gewinne zu einigen. Hierzu sind offene Kommunikation, vertrauensvolle Zusammenarbeit, Fairneß, ein auf Problemlösung gerichteter Verhandlungsstil die Voraussetzungen. Dieser wichtige Punkt wird aufgrund seiner Bedeutung in einem eigenen Kapitel nochmals thematisiert.

An dieser Stelle ist es jedoch wesentlich, auf folgendes Dilemma hinzuweisen: Auf der einen Seite sind Koordinationserfolge nur auf der Grund-

15 Honneth benutzt diesen Begriff, um die Handlungsorientierungen und moralischen Zielsetzungen von Akteuren zu thematisieren, welche die Grundlage der Reproduktion komplexer, moderner Gesellschaften sind. 
lage von vertrauenvollen und kooperativen Orientierungen der Akteure wahrscheinlich. Auf der anderen Seite wird im Verlauf der Verhandlungen und der Festlegung der Lösung über die Verteilung der Kosten und Gewinne und über Fairneßkriterien zu streiten sein. Während die Erzielung eines Ergebnisses ohne kooperative Verhandlungsstile der Beteiligten ausgeschlossen ist, sind im Prozeß der Aushandlung strategisches Handeln, Informationsmanipulierung und der Einsatz von Bluff oder Drohungen wahrscheinlich. Die dem Gesamtergebnis der Verhandlung angemessene konstruktive, auf gemeinsame Problemlösung fixierte Handlungsorientierung kann im Aushandlungsprozeß leicht ausgebeutet werden. Umgekehrt können effektive Bargaining-Strategien, erfolgreiche verhandlungstaktische Manöver, wie Informationsmanipulation, Vorteile im laufenden VerhandlungsprozeB - unter der üblichen Bedingung unvollständiger Information - bedeuten, zugleich aber die Beteiligten letztlich daran hindern, gemeinsame Probleme zu lösen und den insgesamt erreichbaren Kooperationsgewinn zu maximieren. Es ist demnach nicht unwahrscheinlich, daß vor allem Akteure, die darauf ausgerichtet sind, durch kooperative Strategien die Erzielung des »Kaldor-Optimums« zu unterstützen, Gefahr laufen, in der Verteilungsfrage übervorteilt zu werden. In dieser Konstellation besteht das »Verhandlungsdilemma« (Scharpf 1992a; Lax/Sebenius 1986). ${ }^{16}$

\section{Macht in Netzwerkbeziehungen}

»Der Begriff »Macht « ist soziologisch amorph.« Max Weber, Soziologische Grundbegriffe, Tübingen 1960, S. 43. »Das Phänomen der Macht ist einfach und universell, aber der Begriff der Macht ist schwer zu fassen und vielfältig." Crozier/Friedberg, Macht und Organisation, Königstein 1979, S. 39.

Machtphänomene werden in steuerungstheoretisch orientierten Netzwerkanalysen nahezu systematisch ausgeblendet. ${ }^{17}$ Netzwerkstrukturen er-

16 Lax/Sebenius haben dieses Phänomen im Bereich des Unternehmensmanagement untersucht. Scharpf verweist auf die Bedeutung dieses Dilemmas für Verhandlungssysteme und Netzwerke.

17 Hierauf verweist z. B. Mahnkopf (1993). Granovetter (1973 und 1983), einer der Väter der Netzwerktheorie, thematisiert zwar Machtbeziehungen in Netzwerken, jedoch ohne deren Bedeutung für die Funktionsfähigkeit von Netzwerkbeziehungen systematisch zu durchdringen. Die Einschränkung »steuerungstheoretisch orientierten Netzwerkanalysen« ist notwendig, weil in den eher deskritiv ausgerichteten Netzwerkarbeiten durchaus »Macht-Indikatoren« (z. B. Verfügbarkeit über finanzielle Ressourcen) benannt werden, ohne jedoch die Steuerungsfunktionen von Netzwerken genauer zu untersuchen. Vgl. dazu Pappi (1987) und Laumann/Knoke (1987). Waarden (1992) unterscheidet in einer Typologie vier Typen von »distribution of power in Networks und zwar solche, die a) durch einen gegenüber der Gesellschaft autonomen Staat charakterisiert sind, b) durch deu Staat dominiert werden, c) durch starke Interessengruppen dominiert sind sowie d) 
scheinen oft als reibungslos funktionales Zusammenspiel zwischen Akteuren bzw. Akteursgruppen, zur Erreichung eines gemeinsamen Zieles. Für die Ausblendung von Machtbeziehungen in den Netzwerkansätzen gibt es zunächst ein durchaus plausibles Argument, das freilich selten explizit gemacht wird. Netzwerke kommen in der Regel zustanden, weil die Steuerungsressourcen, die zur Lösung eines bestimmten Problemes zu mobilisieren sind, auf eine Vielzahl von Akteuren verteilt sind. Im Netzwerken bestehen also wechselseitige Abhängigkeiten zwischen den Beteiligten, so daß die für die Sozialwissenschaften noch immer als Referenzgrößen dienenden klassischen Macht- und Herrschaftsdefinitionen von Max Weber, die in ihrem Kern auf der Vorstellung von eindeutig identifizierbaren »Machtzentren « basieren, auf Netzwerke kaum Anwendung finden können. Weber schreibt:

»Macht bedeutet jede Chance, innerhalb einer sozialen Beziehung den eigenen Willen auch gegen Widerstreben durchzusetzen, gleichviel worauf diese Chance beruht. Herrschaft soll heißen die Chance, für einen Befehl bestimmten Inhalts bei angebbaren Personen Gehorsam zu finden. « Herrschaft, so Weber, basiert auf Diziplin und Massengehorsam, wobei »Disziplin heißen (soll), die Chance, kraft eingeübter Einstellung für einen Befehl prompten, automatischen und schematischen Gehorsam bei einer angebbaren Vielfalt von Menschen zu finden.« (Weber 1960, 42, 43)

Dieses Verständnis von Macht- und Herrschaft ist nicht ohne weiteres auf Netzwerke übertragbar, weil hier die Steuerungs- und damit Machtressourcen auf unterschiedliche Akteure verteilt sind. Die Durchsetzung von Macht »auch gegen Widerstreben« anderer wird in einem Netzwerksystem, welches durch wechıselseitige Abhängigkeitsstrukturen charkterisiert ist, schwierig, die Ausübung von Herrschaft über Befehle und automatischen Gehorsam ist in hierarchischen Strukturen der Entscheidungsfindung noch denkbar, in Netzwerken ein aussichtloses Unterfangen. Eine Vielzahl von empirischen Arbeiten in ganz unterschiedlichen Politikfeldern, in denen sich netzwerkartige Strukturen herausgebildet haben, belegen dies. Entscheidungen sind oft nur schwer oder gar nicht »gegen Widerstreben« der Betroffenen durchzusetzen. Sind die Entscheidungzentren auf die Kooperation, z. B. die Implementierungsbereitschaft oder -hilfe der Betroffenen, angewiesen, ist die Durchsetzung von Macht im Sinne Webers außerordentlich schwierig. Die Durchsetzung von Entscheidungen bleibt in vielen Fällen auf die Zustimmung der Betroffenen angewiesen. Noch aussichsioser wären Versuche, Herrschaft über »Gehorsam《 und »Disziplin « - im Sinne Webers - durchzusetzen. ${ }^{18}$ Die gleichen Studien verdeutlichen frei-

auf symbiotischen Beziehungen zwischen den Netzwerkakteuren aufbauen. Diese Klassifizierung hat jedoch nur deskriptiven Charakter.

18 So zeigt z. B. Riegraf (1993), daß von Konzernzentralen beschlossene Frauenförderpläne an aktiven wie passiven Formen der Obstruktion (von der Nichtbeachtung, bis zu explizi- 
lich, daß auch in Netzwerkstrukturen Ungleichgewichte existieren, die Handlungsoptionen der Akteure größer oder kleiner ausfallen, einige Akteure also über mehr Macht verfügen als andere. Die Organisationssoziologen Crozier und Friedberg und vor ihnen bereits Weber haben recht, wenn sie betonen, das das Phänomen der Macht oft mit Händen zu greifen scheint, jedoch schwer zu konzeptualisieren oder gar empirisch zu »messen « ist (Crozier/Friedberg 1979, 39ff; Weber 1960, 42ff).

Man könnte angesichts der komplexen Situation in Netzwerken, die durch Machtungleichgewichte, wechselseitige Abhängigkeiten zwischen den Akteuren, bei gleichzeitiger Abwesenheit von Macht und Herrschaft im klassischen Sinne Max Webers gekennzeichnet ist, geneigt sein, Hannah Arendts Terminus der $\gg$ Niemandsherrschaft « aufzugreifen oder einen Buchtitel von Oskar Maria Graf zu zitieren: »Wir sind alle Gefangene«. Die Zurechenbarkeit von Macht - und damit auch Ohnmacht - in Netzwerken ist prinzipiell kompliziert, in hierarchischen Entscheidungsstrukturen hingegen relativ trivial. ${ }^{19}$ Dennoch bleiben die Sichtweisen von Hannah Arendt und Graf wenig aussagekräftig. Einen Ausweg aus dieser Unübersichtlichkeit oder zumindest Orientierungsmarken bieten Crozier und Friedberg, die darauf verweisen, daß »Macht eine gegenseitige, aber unausgewogene Beziehung « ist (Crozier/Friedberg 1979, 40). Diese Sichtweise modifiziert den absoluten Machtbegriff von Weber und erlaubt eine Anwendung auf Interaktionsbeziehungen in Netzwerken. Crozier und Friedberg beschreiben Macht als eine Tauschbeziehung, die auf Gegenseitigkeit beruht, in der aber die Tauschbedingungen einen der beteiligten Akteure begünstigen:

»Es ist ein Kräfteverhältnis, aus dem der eine mehr herausholen kann als der andere, bei dem aber gleichfalls der eine dem anderen nie völlig ausgeliefert ist.« (Crozier/Friedberg 1979, 41)

ten Versuchen der Blockierung) von Teilen der Beschäftigten scheitern können. Ohne eine aktive Beteiligung strategisch wichtiger Akteure ( $z$. B. der Personalentscheider, Abteilungsleiter, Betriebsrïte, Frauenbeauftragten), deren »Gehorsam《 nicht »eingefordert《 werden kann, da sie über Handlungs- und Autonomiespielräume verfügen, die von der Konzernzentrale nicht kontrolliert (»beherrscht«, im Sinne Webers) werden können, ist eine Umsetzung der Programme nahezu unmöglich. Ähnliche Phänomene von Grenzen hierarchischer Steuerung und der wechselseitigen Dependenz der Akteure beschreibt Naschold (1992/1993) für die Abhängigkeit staatlicher Institutionen von der Kooperationsbereitschaft gesellschaftlicher Akteure im Prozeß der Politikfornulierung und Umsetzung, Reichard (1994) am Beispiel der Lokalpolitik, Krumbein (1991) im Kontext regionaler Strukturpolitik, Messner et al. (1991) in der Analyse der Herausbildung von Sektorpolitiken und -institutionen in der chilenischen Industrie, Jürgens et al. (1989) am Beispiel der Umsetzung neuer Produktionskonzepte in der Automobilindustrie.

19 Natürlich können auch zentralistische Entscheidungssysteme durch die Aufkündigung der Folgebereitschaft der $»$ Beherrschten $\ll$ ins Wanken geraten. Dies ist jedoch eher die Ausnahme als die Regel. 
Die Grundlage von Macht ist die Verfügbarkeit über Ressourcen. Unterscheiden lassen sich vier bedeutende Machtquellen und zwar solche,

- die auf der Beherrschung eines spezifischen Sachwissens basieren;

- die sich aus der Kontrolle über Informationen und Kommunikationsquellen ergeben;

- die sich aus dem Vorhandensein allgemeiner organisatorischer Regeln oder auch Wertemustern ableiten lassen;

- die sich aus der Verfügbarkeit über finanzielle Ressourcen ergeben, mit denen zumindest die erste und die zweite Quelle beeinflußbar sind. ${ }^{20}$

Wenn Machtressourcen in Netzwerken in einem solchen Kräftefeld verteilt sind, es neben der Macht der Mächtigen also auch die »Macht der Schwachen« (Crozier/Friedberg 1979, 42) gibt, wodurch werden dann Kräfteverhältnisse und Asymetrien in dem jeweiligen Kräftefeld determiniert? Crozier und Friedberg sprechen vom »Grad der Relevanz der Ressourcen « (ebd., 46), ohne den Begriff der Relevanz genauer zu präzisieren. Sinnvoll erscheint mir eine Unterscheidung in strategisch wichtig und strategisch unbedeutendere Ressourcen. Strategisch wichtig sind Ressourcen, die nicht oder nicht ohne weiteres substituierbar sind, strategisch unbedeutender wären substitujerbare Ressourcen.

Dies hieße z. B. in einem Politiknetzwerk, in dem staatliche Institutionen zur Umsetzung eines Programms auf die Implementierungshilfe intermediärer Institutionen angewiesen sind, folgendes: Wird das Politikprogramm allgemein für wichtig erachtet und die gesellschaftlichen Akteure sind zur Finanzierung des Vorhabens auf den Staat angewiesen, so ergibt sich hieraus bereits die Verfügung über strategisch wichtige Ressourcen durch den Staat. Sollte es nur eine intermediäre Institution geben, die Implementierungshilfe leisten könnte, so verfügt auch diese über strategisch wichtige Machtressourcen. Existieren mehrere Institutionen, zwischen denen der Staat wählen kann, sind deren Machtressourcen geringer, obwohl auch der Staat, aufgrund der Abhängigkeit von der Kooperation mit anderen, nur über eingeschränkte Autonomie verfügt. Entscheidet sich der Staat im zweiten Fall für die Kooperation mit einer Institution, so erhöhen sich im Zeitverlauf die Machtressourcen für letztere, da diese Lerneffekte kumuliert, die aus der Sicht des Staates den Wechsel des Kooperationspartners unattraktiv erscheinen läßt (Transaktionskostenargument). Ein zweites Beispiel: In einem Netzwerk wird wissenschaftlicher Sachverstand benötigt, um eine Problemlösung anzusteuern. Der Wissenschaftler verfügt damit ohne Zweifel über Machtressourcen, auf die die anderen Akteure angewiesen sind. Aber im Normalfall, also unter der Annahme, daß das zur Verfü- 
gung gestellte Know how nicht einmalig, also substituierbar ist, ist der Wissenschaftler austauschbar. Auf ihn kann also größerer Druck ausgeübt werden als auf Akteure, die über nicht oder nur schwerer substituierbare Ressourcen verfügen.

Die Beispiele verdeutlichen, daß es durchaus möglich ist, systematisch über Machtstrukturen in Netzwerken nachzudenken. Die zuweilen, implizit oder explizit, vorhandene Idee, daß in sozialen Beziehungsstrukturen, in denen kein eindeutig identifizierbares Zentrum zu lokalisieren ist, machtfreie Kommunikation oder herrschaftsfreie Beziehungen dominieren, ist demnach naiv. ${ }^{21}$ Machtasymmetrien in Netzwerken implizieren zum einen, daß es auch im Fall von Netzwerksteuerung Gewinner und Verlierer geben kann, und zwar weil sich die relativ Mächtigeren gegen die Schwächeren durchzusetzen vermögen, auch wenn zweifelsohne in Netzwerken, anders als in Strukturen, in denen ein Akteur über alle relevanten Steuerungsressourcen verfügt, die »Macht der Schwachen « zunimmt. Hinter dem Phänomen von Machtstrukturen in nicht hierarchischen Verhandlungssystemen steckt zum anderen ein für unsere Fragestellung zunächst noch relevanteres Problem, das die Funktionsfähigkeit von Netzwerken betrifft. Was in einem Netzwerk als relevante Probleme, adäquate Lösungen und Instrumente sowie vernünftige Zielsysteme definiert wird, hängt einerseits von der Leistungsfähigkeit und Kreativität, also den »kognitiven Möglichkeiten« eines Netzwerkes ab, andererseits jedoch auch von der »Definitionsmacht « der jeweiligen Akteure. Diese dürfte sich primär an der Durchsetzung der eigenen Interessen orientieren. So werden z. B. Chemieunternehmen in einem Politiknetzwerk, in dem es um die Formulierung geeigneter Sektorpolitiken geht, nur in den seltensten Fällen freiwillig auf Umweltargumente eingehen, die ihre Produktionskosten erhöhen könnten oder sie zumindest unter Innovationsdruck setzen.

Netzwerke stehen also trotz ihres interaktiven Charakters nicht per se für »höhere Intelligenz«. Dieser Sachverhalt war schon im Kontext der möglichen sozialen, politischen und kognitiven Blockaden von Netzwerken thematisiert worden. Die Diskussion um Machtstrukturen in Netzwerken führt zu einer weiteren Problemdimension: In Netzwerken können aufgrund der »Definitionsmacht« strategisch wichtiger Akteure wichtige Problemdimensionen ausgeblendet werden. Der gleiche Sachverhalt gilt natürlich auch für die Beziehungen zwischen Netzwerken und ihrer Umwelt. Netzwerke, die sich zu Machtkartellen entwickeln, sind in der Lage, problemlösungsorientierte gesellschaftliche Such- und Lernprozesse zu blockieren

21. In vielen Netzwerkarbeiten wird dieser Zusammenhang zwischen $\gg$ Dezentralisierung und Demokratisierung «, »Enthierarchisierung, horizontale Steuerung und Demokratisierung, Abbau von Macht« unkritisch suggeriert. 
oder zumindest zu verlangsamen sowie Kosten von Anpassungsanforderungen auf ihre Umwelt zu verlagern. ${ }^{22}$

Diesen Problemzusammenhang hat Karl Deutsch $(1963,21)$ in seiner Definition von $»$ Macht besonders betont. Er umschrieb »Macht« als »die Fähigkeit, nicht lernen zu müssen«. Deutsch gelang es mit diesem Machtverständnis, den durchaus ambivalenten Charakter von Macht anzusprechen, der auch in unserem Zusammenhang von Bedeutung ist. Einerseits erlaubt die Verfügbarkeit über Macht, nicht lernen zu müssen, da kein Anpassungsdruck von seiten anderer Akteure besteht. Wer in diesem Sinne über Macht verfügt, befindet sich also in einer zunächst außerordentlich komfortablen Situation, die es, wie bereits gezeigt, eben auch erlaubt, Kosten auf Dritte abzuwälzen oder in die Zukunft zu verlagern. Andererseits sind Machtpositionen, so wie sie Deutsch versteht, stets prekär; insbesondere in gesellschaftlichen Entwicklungssequenzen, in denen »Lernen « zu den wichtigsten Aktivposten zählt und die Verfügbarkeit über »Informationen « (also den Ergebnissen von Lernprozessen) eine bedeutende Machtressource darstellt (Arrow 1972). Wenn Machtpositionen dazu verleiten können, nicht zu lernen und sich an veränderte Bedingungen anzupassen, dann ist in den Grundlagen der Macht potentiell bereits deren Erosion angelegt. ${ }^{23}$ Der Zwang der nicht-Mächtigen, Lernen zu müssen, bedroht beständig die etablierten Machtpositionen. Michael Gorbatschow hat diesen Doppelcharakter der Macht in einer wahrlich historischen Situation, bezogen auf die Lernunfähigkeit des Honecker-Regimes, in einem geflügelten Wort auf den Punkt gebracht: »Wer zu spät kommt, den bestraft das Leben. «

Die Suche nach Mechanismen zur Entwicklung von möglichst problemund nicht einseitig (partikular)interessenorientierten Lösungsmustern ist also mit der »sozialen Innovation« des Netzwerkes, entgegen der Hoffnung vieler Netzwerktheoretiker, keineswegs beendet. ${ }^{24}$ In Netzwerken und zwischen Netzwerken und ihrer Umwelt vollzieht sich ein nicht endender Prozeß, den man in Anlehnung an Axel Honneth als einen »Kampf (der Ak-

22 Ein Beispiel ist das »Ruhrkartell« aus Schwerindustrie, Landesregierung und Gewerkschaften in NRW, das beinahe zwei Dekaden lang in der Lage war, zukunftsorientierte wirtschaftliche Umstrukturierungen in der Region zu verhindern und Lösungen auf Kosten Dritter (der Steuerzahler) durchzusetzen. Vgl. dazu Grabher (1994, 79ff); Voelzkow (1991); Drüke/Burmeister (1991).

23 Dieser Zusammenhang gilt nur »potentiell«, im Sinne einer Risikoposition, weil Deutsch zu Recht von der $»$ Fähigkeit«, nicht der $»$ Notwendigkeit« nicht lernen zu müssen, spricht. "Mächtige«, die trotz geringem Anpassungsdruck rasch lernen, verfügen über gute Chancen, ihre Machtposition auszubauen.

24 Verwiesen werden kann z. B. auf Habermas (1992) oder auch Peters (1993), die Machtund Entscheidungsstrukturen in modernen Gesellschaften im Spannungsfeld »administrativer«, »sozialer « und »kommunikativer « Machtstrukturen rekonstruieren. 
teure) um Anerkennung « (Honneth 1992; auch Honneth 1989, 384ff), ihrer Identität, ihrer Argumente, ihrer Partizipationsberechtigung, beschreiben könnte. In diesem »Kampf um Anerkennung« kann sich die »Definitionsmacht « durchaus neu verteilen, wie z. B. der Erfolg der Umweltbewegungen zeigt, die innerhalb von zwei Dekaden in der Lage waren, ein marginales Politikfeld in ein zentrales gesellschaftliches Thema zu verwandeln. Daß auch einmal erreichte »Anerkennung « wieder erodieren kann, zeigt sich in dem gleichen Politikfeld: In der Wirtschaftskrise Mitte der 90er Jahre und trotz der UNCED-Konferenz in Rio verliert das Umweltthema in der politischen Praxis an Bedeutung. Machtressourcen und Definitionsmacht werden erneut umgeschichtet, die kurzfristigen Sachzwänge gegen die langfristigen Systemimperative durchgesetzt.

\section{Das Spanoungsverhältnis von Konflikt und Kooperation}

Helmut Dubiel verweist zu Recht darauf, daß die Frage, wie soziale Ordnung, gesellschaftliche Strukturbildung und Integration möglich ist, heute weniger kontrovers diskutiert wird, als noch vor wenigen Jahrzehnten (Dubiel 1991). Abgesehen von radikalliberalen oder traditionell marxistischen Ansätzen besteht im Prinzip Einigkeit darüber, daß soziale Ordnung im kulturellen und sozialen Zusammenspiel von Akteuren und gesellschaftlichen Organisationen sowohl tendenziell bewußt gestaltet wird, als auch hinter dem Rücken der Beteiligten entsteht - durch Marktgesetze, politische Machtstrukturen und technisch-ökonomische Imperative in einer integrierten Weltgesellschaft. Netzwerktheoretiker beschreiben Netzwerke oft vor allem deshalb als innovative und leistungsfähige Organisationsmuster, da diese in der Lage seien, die Kreativität der jeweiligen Akteure und Organisationen, die Eigenlogik von Systemen sowie das bewußte Zusammenwirken von Netzwerkteilnehmern zur Erreichung gemeinsamer Ziele zu kombinieren.

Es wurde bereits dargestellt, daß Netzwerke zu ihrer Stabilisierung der Herausbildung einer »Kultur der Kooperation" oder auch des Konsenses und sozialer Kohärenz bedürfen. »Kooperation « ist ein Schlüsselbegriff der Netzwerktheorien; sie soll der Integration von Netzwerken dienen, den in modernen Gesellschaften dominanten Kräften der Desintegration, Fragmentierung und der »Kultur der Separation « (Dubiel 1991, 134) entgegenwirken, zur Stärkung gemeinsamer Interessen in Netzwerken beitragen. Diese Sichtweise leitet sich aus der zunächst richtigen Einsicht ab, daß funktionsfähige Netzwerkstrukturen nicht auf rein kompetitiven oder gar feindlichen Beziehungen zwischen den beteiligten Akteuren aufbauen können. Oft schlägt dieser Diskurs jedoch in eine einseitig harmonistische 
und somit naive Deutung der Funktionsweise von Marktgesellschaften um. Dies gilt z. B. für eine Reihe von Arbeiten zu den »Industriedistrikten « im nördlichen Italien. Lokale und regionale Netzwerke, die zur Herausbildung von »collective efficiency« und Wettbewerbsfähigkeit beitragen, werden nicht selten - mit Verweis auf die sozialen Integrationskräfte von stabilen lokalen Identitäts- und Wertemustern sowie homogenen Sozialstrukturen in den districts - als geradezu idyllische Gegenmodelle zur rauhen, liberalen Marktwirtschaft idealisiert. ${ }^{25}$ Im spannungsgeladenen Weltdorf wächst die Sehnsucht nach überschaubaren Zusammenhängen und möglichst harmonischen Lösungsmustern. In diese Richtung können auch die Appelle der konservativen Kommunitaristen interpretiert werden. Gegen die Konflikthaftigkeit säkularisierter Gesellschaften setzten sie die Homogenität von Gemeinschaften, die durch übereinstimmende Vorstellungen vom »guten Leben« und »gemeinschaftliche Wertemuster « integriert sind. ${ }^{26}$

Netzwerktheorien und Netzwerkpraktiker die dieser einseitig kooperationsorientierten Perspektive folgen, übersehen, daß zu enge Kooperationsbeziehungen in Netzwerken zu sozialen, kognitiven und funktionalen Blokkierungen führen können. »Kooperation « kann sich demnach in interaktiven Prozessen sowohl außerordentlich produktiv, als Entwicklungsmotor, aber eben auch als Blockademechanismus auswirken.

Interessanterweise wird in den Sozialwissenschaften parallel zu den kooperationsorientierten Diskursen eine geradezu entgegengesetzt ausgerichtete Debatte geführt. Auf der Suche nach Institutionen und Handlungsorientierungen, die zur Integration moderner Gesellschaften beitragen können, stößt Dubiel auf eine »Kultur des Konfliktes«. Während Konflikte im allgemeinen als zerstörerisch angesehen werden, und z. B. im Rahmen der Kommunitarismusdebatte nach Mechanismen Ausschau gehalten wird, um diese durch Gemeinsinn einzudämmen, argumentiert Dubiel, daß Bindungen und Zusammenhalt in modernen Gesellschaften geradezu durch gesellschaftliche Konflikte hervorgebracht werden (Dubiel 1991, 1993).

Dubiel lehnt sich explizit an eine demokratietheoretische Diskusssion an, die in den frühen $80 e r$ Jahren in Frankreich geführt wurde. ${ }^{27}$ Im Zentrum steht die These, daß in modernen, demokratischen Gesellschaften Konflikt ein wesentlicher Sozialisationsfaktor und ein eminent wichtiger und effektiver Mechanismus zur Herstellung von Kohärenz und Integration sei. Konflikte produzieren das »soziale Kapital «, das zu ihrer Bewältigung und

25 Die harmoniebetonten Interpretationen werden auch von Nadvi/Schmitz (1994) kritisiert; ähnlich Qualmann (1993).

26 Vgl. die Arbeiten in Honneth (1993). Ein wesentlicher Vertreter des konservativen Kommunitarismus ist Robert Bellah (Bellah et al. 1985).

27 Vgl. zum Überblick den Sammelband von Rödel (1990), insbesondere den Aufsatz von Gauchet, auf den sich Dubiel explizit bezieht. 
für den Zusammenhalt der Gesellschaft notwendig ist. In Abgrenzung zur marxistischen Diskussion bis in die 70er Jahre, in der Konflikte - zuweilen jedweder Art - als antagonistische und die kapitalistischen Gesellschaften letztlich zersetzende Krisen interpretiert wurden, werden Konflikte nun als die eigentlichen Stützen der Gesellschaft entdeckt.

Dubiel übernimmt diese Sichtweise und verknüpft sie mit der deutschen Diskussion. Der »Verfassungspatriotismus« von Habermas, welcher die Bürger auf die Legitimität demokratischer Prozeduren und deren Resultate verpflichten soll, wird ergänzt durch die Idee, Gemeinsinn und gesellschaftliche Integration entstehe eben primär durch gesellschaftliche Konfliktaustragung. Die Gesellschaft produziert einen kontinuierlichen Strom von Konflikten, die zu bearbeiten und zu lösen die Akteure lernen müssen. Im Ergebnis werden permanente gesellschaftliche Such- und Lernprozesse stimuliert. ${ }^{28}$ Die Vitalität und Innovationsfähigkeit pluralistisch-marktwirtschaftliche Gesellschaften bestünde demnach in ihrer Fähigkeit, sich aus sich selbst heraus permanent zu erneuern. Hirschman begründet dieser Argumentationslogik entsprechend $\gg$ den Verfall und den Verlust an Lebensfähigkeit der kommunistisch regierten Gesellschaften mit dem 'Erfolg', mit dem offene gesellschaftliche Konflikte unterdrückt wurden.« (Hirschman 1994, 300)

Auf den prekären und für moderne Gesellschaften konstitutiven Zusammenhang zwischen Konflikt sowie Kooperation und Harmonie hat auch Karl Popper in seiner »Offenen Gesellschaft $<$ hingewiesen. Er warnte davor, daß offene Gesellschaften stets in der Gefahr stünden, sich zu »abstrakten, entpersönlichten Gesellschaften « zu entwicklen, in denen zunehmende Konflikte zwischen gesellschaftlichen Akteuren, Anonymität und Isolation die Individuen als soziale Wesen gefährdeten (Popper 1980, Bd. 1, 235). Aufgrund dieser latenten Bedrohung durch Zerfalls- und Auflösungserscheinungen, tendieren - so Popper - durch die »Offene Ge-

28 Diese Argumentation ist aus der breiten Literatur über positive Effekte von Krisen geläufig. Im entwicklungstheoretischen Kontext hat z. B. Thomas Hurtienne $(1986,1987)$ auf die potentiell auch produktiven Effekte der Verschuldungskrise der Jateinamerikanischen Länder hingewiesen. Hirschman hat in seinen frühen Arbeiten (1958) zur Modernisierung von Gesellschaften - entgegen der später populären, dependenztheoretischen Vorstellungen von der entwicklungshemmenden Wirkung »struktureller Heterogenität - auf die Bedeutung von Ungleichzeitigkeiten, Ungleichgewichten und Konflikten für dynamische Entwicklung hingewiesen. Auch Dahrendorf (1957) beschäftigte sich mit den positiven Funktionen von Konflikt und Krise. Hirschman verweist zu Recht darauf, daß Dubiel und andere auf diese lange Geschichte sozialwissenschaftlicher Forschung über die Funktionen von Krisen und Konflikten in modernern Gesellschaften nicht eingehen. So wird stets »neu《 erfunden, was provokativ bereits bei Heraklit (»Der Krieg ist der Vater aller Dinge «), brutal bei Nietzsche (»Was mich nicht umbringt, macht mich stärker «) und poetisch bei Hölderlin (»Wo aber Gefahr ist, wächst das Rettende auch «) angelegt war (Hirschman 1994). 
sellschaft« überforderte Menschen dazu, sich auf die »geschlossene Gesellschaft« zurïckzuorientieren, in der auf der Grundlage kollektiver und verbindlicher Werte wieder ein »organisches, harmonisches Ganzes« entstehen könne (Popper 1980, Bd. 1, 232ff).

Eine solche Kehrtwende, zurïck zur geschlossenen Gesellschaftsordnung, würde die persönliche Freiheit, Verantwortlichkeit und Kreativität der Individuen zerstören und damit die Entwicklungspotentiale der »Offenen Gesellschaft« unterminieren. Zugleich sei die Hoffnung, durch Harmonisierung und die Überwindung von Konflikt, den Risiken der »Offenen Gesellschaft « begegnen zu können, trügerisch - da sie in gesellschaftlicher Stagnation münde. ${ }^{29}$ Die $» O f f e n e$ Gesellschaft « müsse sich gegenüber diesen beiden Bedrohungen - soziale Desintegration in der konfliktdominierten »abstrakten Gesellschaft« versus Rückbezug auf ganzheitliche Harmonie und geschlossene Kollektive in der organischen Gesellschaft - behaupten. Popper schließt den ersten Band seiner Arbeit mit folgenden Überlegungen:

»Je mehr wir versuchen, zum heroischen Zeitalter der Stammesgemeinschaft (der 'Geschlossenen Gesellschaft', D.M.) zurückzukehren, desto sicherer landen wir bei der Inquisition, ... einem romantisierten Gangstertum (und) ... der Unterdrückung von Vernunft und Wahrheit. ... Wir müssen ins Unbekannte, ins Ungewisse, ins Unsichere weiterschreiten und die Vernunft, die uns gegeben ist, verwenden, um, so gut wir es eben können, für beides zu planen: nicht nur für Sicherheit, sondern zugleich auch für Freiheit« (Popper 1980, Bd. 1, 268).

Wer dächte angesichts dieser Warnungen von Popper nicht an die neokonservativen Fraktionen in der Kommunitarismusdebatte, aber eben auch romantisch verklärte Perzeption von Autoren, die Netzwerke als kooperativ-harmonisches Ganzes idealisieren.

Die Argumentationen von Dubiel, Hirschman und Popper sollten überzogen kooperations- und harmonieorientierte Netzwerktheoretiker ernst nehmen. Die Ausschaltung jeglichen Konfliktpotentials unterminiert nicht nur in Gesamtgesellschaften, sondern auch in Netzwerken deren soziale, politische oder auch ökonomische Innovations- und Reaktionsfähigkeit. Neben diesem quasi »innovationstheoretischen « Argument ist ein weiterer Hinweis wichtig. Die Häufigkeit und Allgegenwart von Konflikten ist augenscheinlich ein wichtiges Kennzeichen pluralistisch-marktwirtschaftlicher Gesellschaften. Marktwirtschaftliche und demokratisch verfaßte Gesellschaften bringen notwendigerweise und in niemals endender Folge Spa-

29 Dabei ist interessant, daß Popper $(1980,232)$ persönliche Verantwortung und Individualismus nicht gegen kollektive Identitäten und Gruppensolidarität ausspielt. Vielmehr stellt die Verantwortung des Individuums für die Folgen seines Handelns, welche andere betreffen, die Grundlage für nicht zerstörerischen Individualismus dar. Diese Überlegungen erinnern nicht zufällig an den kategorischen Imperativ von Kant, dem Popper sein Lebenswerk $\gg$ Die offene Gesellschaft « gewidmet hat. 
nungen und Konflikte hervor, so daß jede Vorstellung von einer permanenten Ordnung und Harmonie unhaltbar ist. Die Hoffnung, Konflikte durch Kooperation neutralisieren zu können, ist demnach a priori ein aussichtloses Unterfangen.

Die Funktion von Netzwerken kann es demnach nicht sein, Konflikte »aufzuheben « und vollständige soziale Kohäsion durch möglichst weitgehende Kooperation anzusteuern, sondern Konflikte handhabbar zu machen. Hirschman betont zu Recht, daß aufgrund der prinizpiell konflikthaften und spannungsgeladenen Strukturen moderner Gesellschaften der Problemlösungsmodus des »Sichdurchwurstelns « außerordentlich erfolgreich sein kann. ${ }^{30}$ Dies gilt auch für Netzwerkstrukturen, die hierzu möglicherweise besonders geeignet sind.

Hirschman verweist jedoch auch darauf, daß Konflikte in Gesellschaften nicht nur als »Klebstoff « sondern eben auch als »Lösungsmittel oder gar »Dynamit« wirken können, die soziale Beziehungen auflösen oder auseinandersprengen (Hirschman 1994, 294). Dies heißt:

- Erstens, daß untersucht werden müßte, unter welchen gesellschaftlichen Bedingungen Konflikte in welche Richtung wirken. Eine allgemeine Antwort kann es hier wohl kaum geben. ${ }^{31}$

- Zweitens, und dies ist im Kontext der zu behandelnden Frage der entscheidende Punkt, gelten all die Argumente gegen eine einseitig kooperations- und harmonieorientierte Sichtweise auf Entscheidungs- und Problemlösungsmechanismen auch für eine überzogene Glorifizierung des produktiven Potentials von Konflikten.

In ausdifferenzierten Gesellschaften im Allgemeinen und Netzwerken im Speziellen wirkt also stets ein Spannungs- und zugleich Abhängigkeitsverhältnis zwischen Konflikt und Kooperation sowie - worauf Popper früh hingewiesen hat - zwischen individueller Freiheit und Kreativität, Gruppenverantwortung und Sicherheit. Auch Fred Hirsch hat zu Recht darauf aufmerksam gemacht, daß gerade in auf Wettbewerb basierenden Marktwirtschaften ein beachtliches Maß an Kooperation und Vertrauen zwischen

30 Hirschman (1994, 301). Hirschman verweist auf den klassischen Aufsatz »The Science of Muddling Through « von Lindblom (1959).

31. Hirschman (1994) unterscheidet zwischen »teilbaren«, »mehr oder weniger - Konflikten« (Verteilungskonflikte), die im Prinzip durch die »Kunst des Kompromisses und Verhandelns« leichter zu lösen sind als »unteilbare«, »entweder oder - Konflikte« (z.B. Streit um multikulturelle Gesellschaft versus homogene Gesellschaft, Konflikt um Abtreibung). Letzterer trägt leicht den Keim dessen in sich, was Hirschman $(1994,304)$ als $\gg$ Lösungsmittel《 oder $\gg$ Dynamit « bezeichnet. Hirschman verweist aber auch darauf, daß oft Konflikte, mit denen Gesellschaften noch nicht umzugehen gelernt haben, zunächst mit dem Etikett »unteilbar, »entweder-oder-Probleme« versehen werden. Hirschman: »Diese Konflikte können nur allmählich verstanden werden, indem wir sie durchleben. « 
den gesellschaftlichen Akteuren Bedingung für funktionsfähigen Wettbewerb in Wirtschaft und Politik darstellt (Hirsch 1977).

Möglicherweise sind gerade Netzwerke, inbesondere im Gegensatz zum Markt, Organisationsformen, deren Stärke in der Chance bestehen könnte, Logiken von "Konflikt und Kooperation « produktiv miteinander in bezug zu setzen. Sicher ist, daß die Leistungsfähigkeit von Netzwerken sowohl durch einseitige Konfliktorientierungen, als auch durch überzogen harmonistische Handlungsstrategien bedroht ist. Wie so oft, haben wir es im Hinblick auf die Funktionen und Folgen von »Kooperation « und »Konflikt« mit einem »Zwischenphänomen« (Narr 1994, 336) zu tun, welches nicht zu einer Seite hin auflösbar bzw. monokausal reduzierbar ist.

\section{Literatur}

Altvater, E. (1991): Die Zukunft des Marktes, Münster.

Altvater, E. (1993): Gewinner und Verlierer, Entwicklung als »Niederauffahrt«, in: Bläter des Informationszentrums Dritte Welt, Heft 191.

Arrow, K. (1972): Gift and Exchanges, in: Philosophy and Public Affairs, Nr. 1.

Ashoff, G. (1988): Rent-Seeking: Zur Relevanz eines relativ neuen Konzeptes in der ökonomischen Theorie der Politik und der entwicklungstheoretischen Diskussion, in: Vierteljahresberichte, $\mathrm{Nr} .112$.

Bellah, R. et al, (1985): Habits of the Heard: Individualism and Commitment in American Life, Berkely.

Benz, A., (1992): Zusammenarbeit zwischen den Norddeutschen Bundesländern, in: A. Benz et al. (Hg.), Horizontale Politikverflechtung, Frankfurt.

Brücker, H. (1993): Privatisierung in Ostdeutschland: Eine institutionenökonomische Analyse, Dissertation am Fachbereich Wirtschaftswissenschaften, Universität Frankfurt.

Coase, R.H. (1960): The problem of social cost, in: Journal of Law and Economics, Nr. 3.

Crozier, M.; Friedberg, E. (1979): Macht und Organisation, Königstein.

Dahrendorf, R. (1957): Soziale Klassen und Klassenkonfikt in der industriellen Gesellschaft, Frankfurt.

Dahrendorf, R. (1992): Der moderne soziale Konflikt, Stuitgart.

Dahrendorf, R. (1992): Die Zukunft der Bürgergesellschaft - Sie ist die entscheidende Säule der Freiheit, in: Frankfurter Rundschau, 20.09.1992.

De Vroey, M. (1991): Der Markt - von wegen einfach, in: PROKLA, Nr. 82.

Deutsch, K. (1963): The Nerves of Government, New York.

Drüke, H.; Burmeister K. (1991): NRW - Das sozialdemokratische Modell einer Industriepolitik?, in: U. Jürgens; W. Krumbein, ( $\mathrm{Hg}$.), Industriepolitische Strategien, Berlin.

Dubiel, H. (1991): Konsens oder Konflikt, Frankfurt.

Dubiel, H. (1993): Das ethische Minimum, in: Siiddeutsche Zeitung vom 27./28. März 1993.

Esser, K.; Hillebrand, W.; Messner, D.;Meyer-Stamer, J. (1993): International Competitiveness in Latin America and East Asia, London.

Esser, K.; Hillebrand, W.; Messner, D.;Meyer-Stamer, J. (1994): Systemische Wetrbewerbsfähigkeit: Internationale Wettbewerbsfahigkeit der Unternehmen und Anforderungen an die Politik, DIE, Berlin.

Giddens, A. (1992): Die Konstitution der Gesellschaft, Frankfurt.

Grabher, G. (1994): Lob der Verschwendung, Berlin.

Granovetter, M. (1973): The Strength of Weak Ties, American Joumal of Sociology, Nr.6.

Granovetter, M. (1983): The Strength of Weak Ties: a Network Theory Revisited, in: R. Collins (Hg.), Sociological Theory, San Francisco.

Habermas, J. (1992): Faktizität und Geltung, Frankfurt. 
Hayek, F.A. (1952): Individualisnus und wirtschaftliche Ordnung, Zürich.

Heritier, A. (1993): Policy-Analyse, in: A. Héritier (Hg.), Policy-Analyse: Kritik und Neuorientierung, Opladen.

Hirsch, F. (1977): Social Limits of Growth, London.

Hirschman, A.O. (1958): The Strategy of Economic Development, Princeton.

Hirschman, A.O. (1994): Wievicl Gemeinsinn braucht die liberale Gesellschaft? in: Leviathan, Nr. 2.

Honneth, A. (1989): Kritik der Macht, Frankfurt.

Honneth, A. (1992): Kampf um Aneikennung, Frankfurt.

Honneth, A. (Hg.) (1993): Kommunitarismus, Frankfurt.

Honneth, A. (1994): Das Andere der Gerechtigkeit, in: Deutsche Zeitschrift für Philosophie, Nr. 2.

Honneth, A. (1994a): Die soziale Dynamik von Mißachtung, in: Leviathan, Nr. 1.

Hurtienne, T. (1986): Fordismustheorie, Entwicklungstheorie und Dritte Welt, in: Peripherie Nr. 22/23.

Hurtienne, T. (1987): Gibt es für den verschuldeten Kapitalismus einen Weg aus der Krise, in: E. Altvater et al. (Hg.), Die Armut der Nationen, Berlin.

Jürgens, U. et al. (1989): Moderne Zeiten in der Automobilindustrie, Berlin.

Kaldor, N. (1939): Welfare Propositions of Economics and Inter-Personal Comparison of Utility, The Economic Journal, Nr. 49.

Kenis, P.; Schneider, V. (1991): Policy Networks and Policy Analysis, in: Marin; Mayntz (Hg.), Policy Networks, Frankfurt.

Kiessling, B. (1988): Kritik der Giddenschen Sozialtheorie, Frankfurt.

Kirsch, G. et al., (1982): Jenseits von Markt und Macht, Baden-Baden.

Knieper, R. (1993): Staat und Nationalstaat, in: PROKLA, Nr. 90.

Krumbein, W. (1991): Industriepolitik: Die Chance einer Integration von Wirtschafts- und Gesellschaftspolitik, in: U. Jürgens, W. Krumbein (Hg.), Industriepolitische Strategien, Berlin.

Kuran, T. (1988): Theories of Personal and Collective Conservatism, in: Journal of Economic Behaviour and Organization, Nr. 10.

Laumann, E.O.; Knoke, D. (1987): The Organizational State, Madison.

LAX, D.A.; Sebenius, J.K. (1986): The Manager as Negotiator, New York.

Leffler-Franke, U. (1994): Stärkung von Partizipation und Selbsthilfe im Zusammenhang mit Strukturanpassung, DIE, Berlin.

Lindblom, C.E. (1959): The Science of Muddling Through, Public Administration Review 19.

Maggi, C. (1994): Decentralización territorial y competitividad: El caso de Chile, Deutsches Institut für Entwicklungspolitik, Berlin.

Mahnkopf, B. (1993): Markt, Hierarchie und soziale Beziehungen, in: N. Beckenbach, W. v. Treeck (Hg.), Umbriiche der gesellschaftlichen Arbeit, Düsseldorf.

Marin, B.; Mayntz, R. (1991): Policy Networks, Frankfurt.

Marmora, L. (1992): Sustainable Development and soziale Gerechtigkeit, in: PROKLA 86.

Marmora, L. (1994): Soziale Gerechtigkeit und Weltmarktintegration am Beispiel Lateinamerika, in: W. Hein (Hg.), Umbruch in der Weltgesellschaft, Hamburg.

Marx, K. (1867ff): Das Kapical, Bd. 1 - 3, in: MEW, 23-25.

Mayntz, R. (1993): Policy-Netzwerke und die Logik von Verhandlungssystemen, in: A. Héritier (Hg.), Policy-Analyse, Opladen.

Mayntz, R.; Neidhardt, F. (1989): Parlamentskultur: Handlungsorientierungen von Bundestagsabgeordneten, in: Zeitschrift für Parlamentsfragen, Nr. 20.

Messner, D. (1993): Stärkung technologischer Kompetenz in Bolivien, Deutsches Institut für Entwicklungspolitik, Berlin.

Messner, D. (1993): Shaping Competitiveness in the Chilean Industry, in: CEPAL Review, Nr. 49 , Santiago.

Nadvi, K.; Schmitz, H. (1994): Industrial Clusters in Less Developed Countries: A Review of Experiences and Research Agenda, Institute of Development Studies, Discussion Paper, Nr. 339, Sussex. 
Narr, W.D. (1994): Recht - Demokratie - Weltgesellschaft, in: PROKLA, Nr. 94.

Naschold, F. (1992): Den Wandel organisieren, Berlin.

Naschold, F. (1993): Organization Development, National Programs in the Context of International Competition, in: F. Naschold et al. (Hg.), Constructing the New Industrial Society, Maastrich.

Nozick, R. (1974): Anarchy, State, and Utopia, New York.

Ohamae, K. (1993): The Rise of the Region State, in: Foreign Affairs, Nr. 2.

Pappi, F.U. (1987): Methoden der Netzwerkanalyse, München.

Peters, B. (1993): Die Integration moderner Gesellschaften, Frankfurt.

Peters, T.J. (1987): Thriving on Chaos: Handbook for a Management Revolution, New York.

Polanyi, K. (1978): The Great Transformation, Frankfurt.

Popper, K. R. (1980): Die offene Gesellschaft und ihre Feinde, München.

Powell, W. W. (1990): Neither Market nor Hierarchy: Network Forms of Organization, in: Research in Organizational Behavior, Nr. 12.

Qualmann, R. (1993): Bedingungen der Wettbewerbsfähigkeit kleiner Unternehmen - Eine kritische Analyse der Konzeption der Industrial Districts, Diplomarbeit am FB Wirtschaftswissenschaft der Universität Tübingen, Tübingen.

Rawls, J. (1971): A Theory of Justice, Harvard.

Rawls, J. (1993): Political Liberalism, New York.

Reichard, C. (1994): Umdenken in Rathaus, Berlin.

Riegraf, B. (1993): Fratenförderung: Alibi oder Aufbruch zur Chancengleichheit, Berlin.

Rödel, U. (1990): Autonome Gesellschaft und Libertäre Demokratie, Frankfurt.

Scharpf, F.W. (1991a): Political Institutions, Decision Styles, and Policy Choices, in: R. Czada, A. Windhoff-Héritier (Hg.), Political Choice, Frankfurt.

Scharpf, F.W. (1991b): Games real Actors could play: The Challenge of Complexity, in: Journal of Theoretical Politics, Nr. 3.

Scharpf, F.W. (1992a): Zur Theorie von Verhandlungssystemen, in: A. Benz et al. (Hg.), Horizontale Politikverflechtung, Frankfurt.

Scharpf, F.W. (1992b): Koordination durch Verhandlungssysteme: Analytische Konzepte und institutionelle Lösungen, in: A. Benz et al. ( $\mathrm{Hg}$.), Horizontale Politikverflechtung, Fft/M.

Scharpf, F.W. (1993a): Positive und negative Koordination in Verhandlungssystemen, in: A. Héritier (Hg.), Policy-Analyse, Opladen.

Scharpf, F.W. (1993c): Coordination in Hierarchies and Networks, in: F.W. Scharpf (Hg.), Games in Hierarchies and Networks, Frankfurt.

Shubik, M. (1987): Game Theory in the Social Sciences, Cambridge.

Selten, R. (1986): Institutional Utilitarianism, in: F.X. Kaufmann et al. (Hg.), Guidance, Control and Evaluation, Berlin.

Semmler, W. (1991): Markt- und nichtmarktförmige Regulierung, in: PROKLA, Nr. 82.

Simon, H. A. (1973): The Organization of Complex Systems, in: H. H. Pattee (Hg.), Hierarchy Theory, The Challenge of Complex Systens, New York.

Simon, K. (1993): Subsidiarität als Rahmenbedingung für Entwicklung, in: K. Simon et al. (Hg.), Subsidiarität in der Entwicklungszusammenarbeit: Dezentralisierung und Verwaltungsreform, Baden-Baden.

Thompson, J.D. (1967): Organizations in Action, New York.

Tugendhat, E. (1993): Vorlesung ïber Ethik, Frankfurt.

Voelzkow, H. (1991): Organisatorisch-institutionelle Aspekte einer regionalen Industriepolitik - illustriert an Beispiel Nordrhein-Westfalen, in: U. Jürgens, W. Krumbein (Hg.), Industriepolitische Strategien, Berlin.

Waarden, F (1992): Dimensions and Types of Policy Networks, in: European Journal of Political Research, Nr. 21.

Weber, M. (1960): Soziologische Grundbegriffe, Tübingen.

Zintl, R. (1991): Kooperation und die Aufteilung des Kooperationsgewinns bei horizontaler Politikverflechtung, Discussion Paper des Max-Planck-Institutes für Gesellschaftsforschung, Köln. 